\title{
Graphitization of amorphous carbons: a comparative study of interatomic potentials
}

\author{
Carla de Tomas \\ Department of Physics and Astronomy, Curtin University, Perth WA 6102, Australia \\ Irene Suarez-Martinez \\ Department of Physics and Astronomy, Curtin University, Perth WA 6102, Australia \\ Nigel A. Marks* \\ Department of Physics and Astronomy, Curtin University, Perth WA 6102, Australia
}

\begin{abstract}
We perform a comparative study of six common carbon interatomic potentials: Tersoff, REBO-II, ReaxFF, EDIP, LCBOP-I and COMB3. To ensure fair comparison, all the potentials are used as implemented in the molecular dynamics package LAMMPS. Using the liquid quenching method we generate amorphous carbons at different densities, and subsequently anneal at high temperature. The amorphous carbon system provides a critical test of the transferability of the potential, while the annealing simulations illustrate the graphitization process and test bond-making and -breaking. A wide spread of behavior is seen across the six potentials, with quantities such as $s p^{2}$ fraction, radial distribution function, morphology, ring statistics, and 002 reflection intensity differing considerably. While none of the potentials is perfect, some perform particularly poorly. The lack of transferability can be traced to the details of the functional form, suggesting future directions in the development of carbon potentials.
\end{abstract}

\section{Introduction}

When molecular dynamics (MD) was developed in the late 1950's to study radiation effects, the metals were easily described. Indeed, the very first MD simulation by Gibson in 1960 [1], an analysis of focussed collision sequences in copper, has a precision that is still relevant today. Metals were a textbook case for the construction of an interatomic potential, with even the simplest Lennard-Jones form providing useful predictions. Progress in developing potentials for these systems advanced quickly, and by the time embedded atom methods $[2,3]$ were developed in the mid 80 's, much of the complexity of metallic bonding had been captured.

Carbon, on the other hand, has proved difficult to study computationally. The two main reasons are the complexity of the competing hybridizations and long-range effects associated with $\pi$-electrons. The breakthrough came in 1986 when Tersoff developed a potential for silicon [4], and two years later, a related potential was released for carbon [5]. The Tersoff potential was quickly adopted by the carbon community interested in studying newly discovered forms such as fullerenes, nanotubes and tetrahedral amorphous carbon. All of these systems posed numerous questions, and this novel computational tool was quickly

\footnotetext{
${ }^{*}$ Corresponding author. Tel: 0061 89266-1386. E-mail: N.Marks@curtin.edu.au (Nigel Marks)
} 
applied. When Brenner built upon the Tersoff method to create the hydrocarbon-oriented Reactive Empirical Bond-Order (REBO) potential in 1990 [6], many of the foundations of modern computational carbon modelling had been laid. Today, these two pioneering articles have an impressive footprint, covering nearly 4000 citations between them, the balance in favour of REBO by slightly more than $2: 1$.

Despite their substantial impact, the original Tersoff and REBO potentials did not provide a complete description of carbon. Neither potential included long-range van der Waals terms and some fundamental properties were incorrect, such as the density of graphite and melting point. Both potentials proceeded to evolve over time; Tersoff released a minor change in 1990 [7], and separate authors added a long-range extension in 1996 [8]. Similar developments occurred for REBO, including a long-range extension in 2000 known as AIREBO [9], and a second-generation REBO-II form (without long-range terms) in 2002 [10].

Many other potentials have subsequently been developed, often directed towards a specific application or niche. At the present time, nearly 40 different carbon potentials (see Table 1) can be identified in the literature, some suited only to carbon, while others also treat interactions with other species such as hydrogen, oxygen and silicon. Many of these potentials have not been made publicly available to the computational community, and only some have been coded into freely accessible packages such as LAMMPS [11], DLPOLY [12] or GULP [13]. In recent years LAMMPS has emerged as the default MD package in materials science and for many carbon scientists if a potential is not in LAMMPS it may as well not exist. While LAMMPS has made the life of a computational researcher easier due to its accessibility and high level of parallelization, the transferability of the underlying potential is often not questioned. This opens the risk that a potential will be applied to a system where the potential itself is unsuitable.

In this work we use LAMMPS to study the transferability of six common carbon potentials: Tersoff, REBO-II, ReaxFF, EDIP, LCBOP-I and COMB3. Due to ongoing debugging, we were unable to use the AIREBO routines available in LAMMPS. All the potentials are used as implemented in LAMMPS, with no modifications, and all of the simulations follow the same general protocol. Using the liquid quenching method we generate amorphous carbons at different densities, and subsequently anneal at high temperature. This approach evaluates the transferability of the potential in two ways; (i) the amorphous carbon structures test whether the potential can reproduce the experimental relation between $s p^{3}$ fraction and density, and (ii) the annealed carbons test the ability of the potential to describe graphitization, in which a high $s p^{2}$ fraction and interplanar ordering is expected at low density [50].

The manuscript is structured as follows. Section 2 summarizes the features of the potentials employed, in particular the underlying chemical principles embodied in the functional form. Section 3 presents the practicalities of the simulation methodology. Since the potentials have a range of melting points, the temperatures used during liquid equilibration and long-time annealing need to be tuned according to the potential and density. The results in Section 4 first consider amorphous carbon, followed by a more detailed analysis of the annealed structures. A discussion of the results in relation to the functional form of each potential is presented in Section 5 .

\section{Interatomic Potentials}

The long list of carbon potentials in Table 1 demonstrates the substantial demand for accurate functional forms that model carbon with an acceptable computational cost. While ab initio and tight-binding method provide a high-level of treatment, the calculations are computationally expensive and usually scale as $O\left(\mathrm{~N}^{3}\right)$ due to matrix diagonalization [51]. In contrast, there are numerous applications in carbon science involving large number of atoms and/or long periods of time. For these situations, an analytic potential functional form that is reasonably efficient is essential. The potentials used in this work are those most commonly used to simulate solid carbon phases with LAMMPS. It is instructive to consider the strengths and limitations of each potential, as this affects the transferability of the potential when applied to a range of scientific questions.

The Tersoff potential [5] is the simplest and fastest of the carbon potentials and is widely available. Based on an elegant bond-order treatment, it modifies the strengths of the bonds according to the number of neighbours. The cutoff function used to count neighbours terminates at the comparatively short distance 
Table 1: Summary of carbon potentials detailing their main characteristics, capacity to describe other chemical species and availability in LAMMPS. The potentials are grouped by families and arranged in chronological order. Where relevant the historical basis of the potential is specified. An asterisk in the final column indicates that an additional step is required, such as editing source files or requesting routines/parameters from authors. Acronyms: REBO Reactive Empirical Bond Order; AIREBO Adaptive Intermolecular REBO; SED-AIREBO Screened Environment Dependent AIREBO; ABOP Analytic bond-order potential; EDIP Environment-dependent interaction potential; ReaxFF Reactive Force Field; LCBOP Long-range Carbon Bond Order Potential; MEAM Modified Embedded Atom Method; RSS Reaction State Summation; COMB Charge Optimized Many Body; SW Stillinger-Weber.

\begin{tabular}{|c|c|c|c|c|c|c|}
\hline Family & $\begin{array}{l}\text { Name of } \\
\text { potential }\end{array}$ & Year & Main characteristic & Builds on & $\begin{array}{l}\text { Other } \\
\text { species }\end{array}$ & Ref. \\
\hline \multirow[t]{8}{*}{ Tersoff } & Tersoff & 1988 & Reparametrization for carbon & Si Tersoff & & {$[5]$} \\
\hline & Tersoff & 1989 & Generalization to multicomponent systems & Si Tersoff & $\mathrm{Si}$ & {$[14]$} \\
\hline & Tersoff & 1990 & Reparametrization to treat $\mathrm{C}$ defects in $\mathrm{Si}$ & Tersoff 1989 & $\mathrm{Si}$ & {$[7]$} \\
\hline & Tersoff & 1994 & Minor change in parameters & Tersoff 1990 & $\mathrm{Si}$ & {$[15]$} \\
\hline & Tersoff & 1995 & Variable cutoff rescaled with system volume & Tersoff 1989 & $\mathrm{Si}$ & {$[16]$} \\
\hline & Nordlund & 1996 & Adds van der Waals and uses larger cutoff & Tersoff 1988 & & {$[8]$} \\
\hline & Tersoff & 1998 & Adds nitrogen and boron. & Tersoff 1989 & $\mathrm{Si} / \mathrm{B} / \mathrm{N}$ & {$[17,18]$} \\
\hline & Tersoff & 2005 & Extended cutoff & Tersoff 1988 & & {$[19]$} \\
\hline \multirow[t]{13}{*}{ REBO } & REBO & 1990 & Adds hydrogen. Treats carbon radicals. & Tersoff & $\mathrm{H}$ & {$[6]$} \\
\hline & REBO-II & 2002 & Adds dihedral. New short-range treatment. & REBO & $\mathrm{H}$ & {$[10]$} \\
\hline & AIREBO & 2000 & Adds van der Waals (Lennard-Jones) & REBO-II & $\mathrm{H}$ & {$[9]$} \\
\hline & AIREBO-II & 2002 & Minor change in two parameters & AIREBO & $\mathrm{H}$ & {$[20]$} \\
\hline & REBO-CHO & 2004 & Adds oxygen & REBO-II & $\mathrm{H} / \mathrm{O}$ & {$[21]$} \\
\hline & AIREBO & 2008 & Environment-dependent van der Waals & AIREBO & $\mathrm{H}$ & {$[22]$} \\
\hline & Pastewka & 2008 & Environment-dependent screening function & REBO-II & $\mathrm{H}$ & {$[23]$} \\
\hline & qAIREBO & 2012 & Adds oxygen and charge equilibration & AIREBO & $\mathrm{H} / \mathrm{O}$ & {$[24]$} \\
\hline & SED-REBO & 2013 & Environment-dependent screening function & REBO-II & & {$[25]$} \\
\hline & AIREBO-M & 2015 & Replaces Lennard-Jones with Morse & AIREBO & $\mathrm{H}$ & {$[26]$} \\
\hline & Heggie & 1991 & Wigner-Seitz treatment of local bonding & Tersoff 1988 & & {$[27]$} \\
\hline & Andribet & 1996 & Simpler functional form. More efficient. & Heggie & & {$[28]$} \\
\hline & Broughton & 1999 & Valence bond treatment. No angular terms. & Tersoff 1988 & & {$[29]$} \\
\hline \multirow[t]{3}{*}{ ABOP } & Pettifor & 1999 & Analytic approximation of tight-binding & & $\mathrm{H}$ & {$[30,31]$} \\
\hline & Mrovec & 2007 & Improved $\sigma$ bond term & Pettifor & $\mathrm{H}$ & {$[32]$} \\
\hline & Zhou & 2015 & Reparametrization for pure carbon & Pettifor & & {$[33]$} \\
\hline EDIP & EDIP & 2000 & Long-range $\pi$-repulsion. Dihedral terms. & Si EDIP & & {$[34]$} \\
\hline \multirow[t]{4}{*}{ ReaxFF } & ReaxFF & 2001 & Flexible and general functional form & & $\mathrm{H}$ & {$[35]$} \\
\hline & $\mathrm{ReaxFF}_{\mathrm{CHO}}$ & 2008 & Adds oxygen & ReaxFF & $\mathrm{H} / \mathrm{O}$ & {$[36]$} \\
\hline & ReaxFF-lg & 2011 & Adds low gradient London dispersion & ReaxFF & $\mathrm{H} / \mathrm{O} / \mathrm{N}$ & {$[37]$} \\
\hline & $\operatorname{ReaxFF}_{\mathrm{C} 2013}$ & 2015 & Reparametrization for solid carbon & ReaxFF $_{\mathrm{CHO}}$ & $\mathrm{H} / \mathrm{O}$ & {$[38]$} \\
\hline \multirow[t]{4}{*}{ LCBOP } & LCBOP-I & 2003 & Simultaneous bond-order \& long-range fit & REBO & & {$[39]$} \\
\hline & LCBOP-I+ & 2004 & Adds torsional term & LCBOP-I & & {$[40]$} \\
\hline & LCBOP-II & 2005 & Improved bond dissociation energetics & LCBOP-I+ & & {$[41,42]$} \\
\hline & Erhart/Albe & 2005 & Hybrid Tersoff/REBO. Reparametrization. & Tersoff/REBO & $\mathrm{Si}$ & {$[43]$} \\
\hline \multirow[t]{5}{*}{ MEAM } & Lee/Lee & 2005 & Reparametrized for C. Long-range term. & MEAM & & {$[44]$} \\
\hline & Uddin & 2010 & Developed for Ni catalysis of nanotubes. & MEAM & $\mathrm{Ni}$ & {$[45]$} \\
\hline & Vashishta & 2007 & Covalent \& ionic. Dipoles. Van der Waals. & Si SW & $\mathrm{Si}$ & {$[46]$} \\
\hline & RSS & 2008 & Short-range interactions. No $\mathrm{sp}^{3}$ bonds. & $\mathrm{RSS}$ & & {$[47]$} \\
\hline & Kumagai & 2009 & Screening function and dihedral term. & REBO & & {$[48]$} \\
\hline COMB & COMB3 & 2013 & Charge dependent short-range interactions & REBO-II & $\mathrm{H} / \mathrm{O} / \mathrm{N}$ & {$[49]$} \\
\hline
\end{tabular}


of $2.1 \AA$, an arbitrary choice intermediate between the first and second neighbours. The shape of the cutoff function was not fitted to any experimental or computational data, and follows a cosine shape between 1.8 and $2.1 \AA$. The choice of short cutoff brings significant performance advantages as the calculation of the potential energy involves relatively few atoms. On the other hand, the absence of any interaction beyond $2.1 \AA$ means there is no attraction nor repulsion between graphene layers. This has dramatic consequences for the predicted density of graphite. Experimentally, graphite is much less dense than diamond, but with the Tersoff potential both materials have densities of about $3.5 \mathrm{~g} / \mathrm{cc}$. Computed crystalline properties of these carbon allotropes can be found in Appendix I. The cutoff function also has consequences for the making and breaking of bonds, as the shape and position of this function controls the activation barrier when changing hybridization. This arbitrary choice of cutoff is likely the explanation for why the melting point of carbon with the Tersoff potential is around $6000 \mathrm{~K}$ [5] as compared to the experimental value of around $4300 \mathrm{~K}$. Nordlund et al. [8] made two significant improvements to the Tersoff potential, adding a long-range attractive term and increasing the cutoff to $2.46 \AA$. These two alterations provide a much improved description of the energy barrier to convert graphite into diamond, and in principle would be useful for many different carbon systems. However, this potential is not available in LAMMPS.

The original Reactive Empirical Bond-Order (REBO) potential [6] was an extension of the Tersoff formulation, adding hydrogen and improving the description of radicals. The second generation, known as REBO-II [10], was released twelve years later with a better description of short-range bonding. This was achieved by modifying the functional form, using an improved database for the fitting of parameters and adding a dihedral term to penalise rotation of $\pi$-bonds. In both of these potentials the cutoff function is the same as Tersoff, except that the cosine function transitions between 1.7 and $2.0 \AA$. Accordingly, REBO and REBO-II also encounter problems with the density of graphite. The relationship of graphite/diamond density is inverted as REBO-II potential predicts graphite $3 \%$ more dense than diamond. A long-range extension, known as Adaptive Intermolecular REBO (AIREBO) was released by Stuart et al. in 2000 [9]. Although published two years earlier than REBO-II, the AIREBO function is based on the second generation REBO. The long-range interaction is described using a Lennard-Jones form, combined with a switching function to deactivate the long-range term at short distances. As indicated in Table 1, a significant number of REBO variants have been developed. This includes the addition of oxygen [21], the use of an environmentdependent screening function to improve the description of bond making and breaking [23, 25] and the inclusion of charge-equilibration to describe polar bonds [24]. We originally intended to include AIREBO in this study, but extensive testing of the routines in LAMMPS revealed bugs which affect the ability to equilibrate the liquid and eventually result in the code crashing. At present, the current implementation is not ready to be used for situations involving bond making and breaking. 1

The Environment Dependent Interaction Potential (EDIP) method for carbon [34, 52] is not related to the Tersoff or REBO methods, and is instead based upon an earlier EDIP method for silicon [53, 54]. EDIP for carbon was developed to simulate the growth of amorphous carbon thin films where there is a competition between $s p^{2}$ and $s p^{3}$ hybridization. Accordingly, EDIP treats the cutoff in a very different way to the Tersoff and REBO potentials, using a generalized coordination term to capture long range $\pi$ repulsion and dihedral rotation penalties. Close attention was paid to correctly predicting the energetics of bond making and breaking by fitting to ab initio data for the graphite-to-diamond transition. Due to this treatment of the cutoff, the EDIP potential is relatively long-ranged, extending to $3.2 \AA$. Beyond this point there is no interaction, and hence van der Waals attraction between graphitic layers are not described. Despite this absence, EDIP simulations have proved capable of producing layered structures, [55, 56, 57] as in many situations the driving force for layering is the long-ranged $\pi$-repulsion between $s p^{2}$-bonded layers. One minor omission in EDIP is the absence of an energy penalty for isolated $s p^{2}$ bonds; this occurs due to the use of an atom-centred bond-order which is inherited from the parent model for silicon.

The Reactive Force Field (ReaxFF) potential [35, 58] was originally developed for hydrocarbons and has since been expanded to describe a large number of atomic species including oxygen, nitrogen, sulfur and many metals. The development philosophy of ReaxFF differs significantly to most carbon potentials.

${ }^{1}$ LAMMPS mailing list, http://lammps.sandia.gov/ threads/msg60863.html. 
Instead of using chemical intuition to select a functional form (see [59] for a discussion of this philosophy), the developers of ReaxFF started with a completely general form for the potential which was parametrized against a large data set. Ingredients of the potential encompass many chemical possibilities including bond order, charge transfer, van der Waals, under/over-coordination and torsion. While the ReaxFF family has had significant success across topics as diverse as proteins, fuel cells and catalysis, the performance for solid carbon phases was poor. As an example, the bulk modulus of diamond with the original ReaxFF potential was around 100 times the experimental value. To address this problem, an improved parameter set of ReaxFF for solid carbon phase was released in 2015 [38]. This parameter set, known as $R_{e a x F} F_{C-2013}$, is available on request from the authors, and is compatible with the functional form in LAMMPS [60]. All ReaxFF calculations performed in this work use this updated parametrization.

The Long-range Carbon Bond-Order Potential (LCBOP) [39] is conceptually similar to AIREBO, combining a bond-order description with long-range terms. However, whereas AIREBO was developed by grafting the long-range extension on top of the pre-existing REBO, LCBOP was developed in an integrated manner in which the bond-order terms were developed along with the long-range extensions. This superior form of parametrization avoids problems with switching functions. LCBOP was developed with solid carbon phases in mind, and two major versions have been published. The first version (LCBOP-I) uses a short-range coordination cutoff of $2.2 \AA$, similar to that of Tersoff and REBO, with the drawback that bond dissociation occurs too abruptly due to the rapid fall-off of the cutoff. The second version (LCBOP-II) [41, 42] offers a much-improved description of the energetics of bond breaking by the inclusion of a medium-range interaction between 1.7 and $4 \AA$. It has been successfully applied to a range of challenging systems such as the liquid state [42, 61], the triple point of carbon [42] and ripples in graphene [62]. Unfortunately LCBOP-II is not available in LAMMPS, and hence LCBOP-I is used in this work.

The Charge-Optimized-Many-Body (COMB) family of potentials [49] were originally developed for metal and oxide systems, and in the third-generation (COMB3) [63] has been expanded to include carbon. Like ReaxFF, the COMB approach uses charge-equilibration combined with bond-order terms, and has the flexibility to describe many different atomic species, including metals such as $\mathrm{Cu}$ and Zn. One practical advantage of the COMB family over ReaxFF is speed, as ReaxFF calculations incur a high computational cost. The pure carbon terms in COMB3 were fitted in a stepwise manner, using diamond, graphite and a range of other symmetric structures. To-date, most COMB3 simulations involving carbon have been applications to hydrocarbon and graphene absorption onto metallic surfaces.

\section{Methodology}

For each potential, four MD simulations with densities of 1.5, 2.0, 2.5 and $3.0 \mathrm{~g} / \mathrm{cc}$ are performed in an NVT ensemble. The same general protocol is applied to all simulations and involves three steps: (i) the creation and equilibration of the liquid, (iii) the quenching of the liquid to produce an amorphous solid and (iii) the annealing of the amorphous solid to induce graphitization. An example of the temperature profile throughout this protocol is shown in Figure 1 . Note that the values of $T_{\text {liquid }}$ and $T_{\text {anneal }}$ are specific to the combination of the potential and density as discussed below.

The liquid is created starting from a slightly randomized simple cubic lattice at the desired density. A supercell of $32 \times 32 \times 32$ carbon atoms (i.e. 32,768 atoms) is used, and the initial temperature is $100 \mathrm{~K}$. This structure is highly unstable and immediately collapses and melts, leading to a rapid increase in temperature. For the Tersoff, REBO and EDIP potentials the initial stage of melting is followed in the NVE ensemble, and after $0.25 \mathrm{ps}$ a thermostat is activated to equilibrate the liquid at the desired temperature $\mathrm{T}_{\text {liquid }}$. For ReaxFF, LCBOP-I and COMB3, the thermostat is applied from the beginning as otherwise the temperature becomes too high and the simulation becomes numerically unstable. To ensure the liquid is highly diffusive, the value of $\mathrm{T}_{\text {liquid }}$ (see Table 2) is chosen such that the mean-squared-displacement (MSD) increases linearly with time. A value of $6000 \mathrm{~K}$ is sufficient for REBO-II, EDIP, ReaxFF and COMB3, but a higher temperature of $8000 \mathrm{~K}$ is required for Tersoff and LCBOP-I. These high temperature are not surprising, as the melting point of the Tersoff potential is approximately $1700 \mathrm{~K}$ higher than the experimental value [5], and similar values in the range of 5500-6000 K were reported for the LCBOP family [42]. The liquid is maintained at 
the chosen temperature $T_{\text {liquid }}$ until the potential energy equilibrates. In all cases the liquid is equilibrated for $5 \mathrm{ps}$, except in the case of LCBOP-I which requires an equilibration of $15 \mathrm{ps.}$

The second step involves the quenching of the liquid to form an amorphous carbon network [64]. The liquid is rapidly cooled down to room temperature $(300 \mathrm{~K})$ in 1 ps to produce a solid with the same density as the precursor liquid. The solid is then equilibrated for 4 ps at $300 \mathrm{~K}$, and analyzed prior to the commencement of annealing.

In the final step, the amorphous carbon solids are annealed for a long time (up to $400 \mathrm{ps}$ ) at a temperature, $\mathrm{T}_{\text {anneal, }}$, which is sufficiently high to allow the structure to evolve, but not so high that it melts. The value of $\mathrm{T}_{\text {anneal }}$ has to be chosen with more care than $\mathrm{T}_{\text {liquid, }}$, since the melting point for each amorphous solid is density and potential dependent. To address this problem, we performed a large number of trial simulations and calculated the MSD to quantify diffusion. If the MSD is very low, there is very little atomic rearrangement and a higher temperature is required. On the other hand, if the MSD increases linearly then the temperature is too high, and the structure is molten. Accordingly, we chose an annealing temperature such that the MSD fits between an empirically determined upper and a lower threshold for the first 100 ps. For the low density samples, 1.5 and $2.0 \mathrm{~g} / \mathrm{cc}$, we chose a lower MSD threshold of $20 \AA^{2}$ and an upper MSD threshold of $100 \AA^{2}$. With these values, the atoms have moved (on average) between 4.5 and $10 \AA$ from their original positions. Since the width of a graphitic hexagon is $2.46 \AA$, these displacements are equivalent to roughly 2 and 4 hexagons, respectively. In the case of the high densities, 2.5 and $3.0 \mathrm{~g} / \mathrm{cc}$, lower thresholds must be used, otherwise the structure becomes molten. We chose a lower MSD threshold of $3 \AA^{2}$ (equivalent to 0.7 hexagons) and an upper MSD threshold of $10 \AA^{2}$ (equivalent to 1.3 hexagons). A summary of the values of $\mathrm{T}_{\text {anneal }}$ for each potential and density is presented in Table 2.

All simulations were performed using LAMMPS [11] using periodic boundary conditions. Thermostatting is performed using the canonical-sampling-velocity-rescaling method of Bussi et al. [65] with a relaxation constant of $0.1 \mathrm{ps}$. The timestep has been carefully chosen for each case to ensure energy conservation. The creation of the liquid requires a relatively small timestep of $0.05 \mathrm{fs}$, with the exception of LCBOP-I which needs 0.01 fs. A larger timestep can be used for the annealing simulations. Here, all simulations use 0.2 fs, except for ReaxFF where the timestep is $0.1 \mathrm{fs}$. The computational cost for each potential is summarized in Appendix II.

Energy minimization using a conjugate gradient scheme was performed prior to all structural analysis. This includes snapshots, time evolution of the coordination fractions, ring statistics, radial distribution functions and the Debye diffraction intensity. Coordination numbers were measured by counting neighbours within a cutoff of $1.85 \AA$ A. Shortest-path ring statistics were calculated using the algorithm of Franzblau [66],

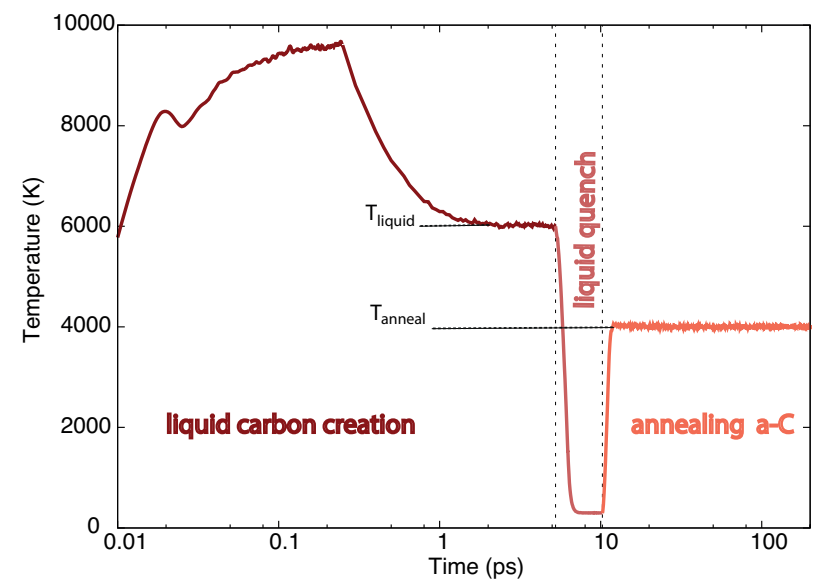

Figure 1: Example of the temperature profile during the creation of the amorphous structure and annealing. The graph shows a semilog plot of the temperature versus time indicating three stages in the simulation protocol: (i) collapse of a randomized simple cubic lattice and equilibration of the liquid at $\mathrm{T}_{\text {liquid, }}$, (ii) rapid quenching of the liquid to produce an amorphous solid followed by equilibration at room temperature, and (iii) annealing of the amorphous carbon at $\mathrm{T}_{\text {anneal }}$. 
Table 2: Simulation temperatures used for equilibration of the liquid and the annealing of the amorphous carbons. For the latter, most potentials require a higher annealing temperature for low density structures.

\begin{tabular}{lccc} 
Potential & $\mathrm{T}_{\text {liquid }}(\mathrm{K})$ & \multicolumn{2}{c}{$\mathrm{T}_{\text {anneal }}(\mathrm{K})$} \\
\cline { 3 - 4 } & & $1.5 \& 2.0 \mathrm{~g} / \mathrm{cc}$ & $2.5 \mathrm{\&} 3.0 \mathrm{~g} / \mathrm{cc}$ \\
\hline Tersoff & 8000 & 5000 & 4500 \\
\hline REBO-II & 6000 & 4500 & 4000 \\
\hline EDIP & 6000 & 4000 & 3000 \\
\hline ReaxFF & 6000 & 3500 & 3000 \\
\hline LCBOP-I & 8000 & 5500 & 5000 \\
\hline COMB3 & 6000 & 4000 & 4000
\end{tabular}

Table 3: Percentage coordination fractions for carbon structures generated using all six potentials and four densities. Data is shown for amorphous carbon $(a-C)$ prepared by liquid quenching and subsequently annealed carbons at the half-way point (200 ps) and at completion (400 ps). The melting and annealing temperatures used are given in Table 2.

\begin{tabular}{|c|c|c|c|c|c|c|c|c|c|c|c|c|c|}
\hline & & \multicolumn{3}{|c|}{$1.5 \mathrm{~g} / \mathrm{cc}$} & \multicolumn{3}{|c|}{$2.0 \mathrm{~g} / \mathrm{cc}$} & \multicolumn{3}{|c|}{$2.5 \mathrm{~g} / \mathrm{cc}$} & \multicolumn{3}{|c|}{$3.0 \mathrm{~g} / \mathrm{cc}$} \\
\hline & & $s p$ & $s p^{2}$ & $s p^{3}$ & $s p$ & $s p^{2}$ & $s p^{3}$ & $s p$ & $s p^{2}$ & $s p^{3}$ & $s p$ & $s p^{2}$ & $s p^{3}$ \\
\hline \multirow{3}{*}{ Tersoff } & $a-\mathrm{C}$ & 23.9 & 71.6 & 4.1 & 12.1 & 78.9 & 9.0 & 4.4 & 76.8 & 18.8 & 1.6 & 64.1 & 34.1 \\
\hline & $200 \mathrm{ps}$ & 11.0 & 84.8 & 4.2 & 8.2 & 85.7 & 6.1 & 2.4 & 81.5 & 16.1 & 0.9 & 65.0 & 34.1 \\
\hline & $400 \mathrm{ps}$ & 9.9 & 85.4 & 4.7 & 8.3 & 85.9 & 5.8 & 2.6 & 81.4 & 16.0 & 1.0 & 65.3 & 33.7 \\
\hline \multirow{3}{*}{ REBO-II } & $a-\mathrm{C}$ & 18.4 & 77.1 & 4.2 & 9.4 & 84.0 & 6.6 & 3.3 & 86.5 & 10.2 & 1.3 & 85.0 & 13.7 \\
\hline & $200 \mathrm{ps}$ & 2.9 & 92.6 & 4.5 & 4.1 & 89.3 & 6.6 & 1.7 & 89.3 & 9.0 & 0.7 & 90.7 & 8.6 \\
\hline & $400 \mathrm{ps}$ & 2.1 & 93.8 & 4.1 & 3.6 & 89.6 & 6.7 & 1.4 & 90.0 & 8.6 & 0.5 & 93.3 & 6.2 \\
\hline \multirow{3}{*}{ EDIP } & $a-\mathrm{C}$ & 29.6 & 62.0 & 8.4 & 15.0 & 66.3 & 18.7 & 4.0 & 60.3 & 35.7 & 0.3 & 44.8 & 54.9 \\
\hline & $200 \mathrm{ps}$ & 3.7 & 93.9 & 2.4 & 2.5 & 93.9 & 3.6 & 0.9 & 62.8 & 36.3 & 0.2 & 39.6 & 60.2 \\
\hline & $400 \mathrm{ps}$ & 2.8 & 95.5 & 1.7 & 2.3 & 95.0 & 2.7 & 0.7 & 65.0 & 34.3 & 0.2 & 39.3 & 60.6 \\
\hline \multirow{3}{*}{ LCBOP-I } & $a-\mathrm{C}$ & 36.6 & 62.7 & 0.1 & 13.0 & 86.5 & 0.5 & 4.2 & 94.0 & 1.8 & 1.0 & 92.7 & 6.3 \\
\hline & $200 \mathrm{ps}$ & 24.5 & 74.9 & 0.2 & 8.8 & 90.8 & 0.4 & 1.4 & 97.9 & 0.7 & 0.6 & 96.9 & 2.5 \\
\hline & $400 \mathrm{ps}$ & 23.2 & 76.2 & 0.2 & 9.0 & 90.6 & 0.4 & 1.4 & 97.9 & 0.7 & 0.6 & 97.0 & 2.4 \\
\hline \multirow{3}{*}{ ReaxFF } & $a-\mathrm{C}$ & 37.7 & 60.5 & 1.7 & 20.3 & 75.1 & 4.3 & 7.8 & 81.2 & 11.0 & 1.5 & 72.1 & 26.4 \\
\hline & $200 \mathrm{ps}$ & 3.6 & 95.2 & 1.2 & 3.5 & 94.5 & 2.0 & 1.1 & 94.4 & 4.5 & 0.2 & 86.0 & 13.8 \\
\hline & $400 \mathrm{ps}$ & 1.9 & 97.1 & 1.0 & 1.8 & 97.1 & 1.1 & 0.7 & 96.0 & 3.3 & 0.1 & 90.2 & 9.7 \\
\hline \multirow[b]{2}{*}{ COMB3 } & $a-\mathrm{C}$ & 18.0 & 58.4 & 22.5 & 14.3 & 55.4 & 29.4 & 9.8 & 52.3 & 36.9 & 6.0 & 49.2 & 43.7 \\
\hline & $200 \mathrm{ps}$ & 14.3 & 51.0 & 33.2 & 10.6 & 49.4 & 39.5 & 6.6 & 49.8 & 43.2 & 3.6 & 48.8 & 47.6 \\
\hline
\end{tabular}

using an in-house code previously used to study amorphous carbon [67]. The same cutoff of $1.85 \AA$ was used to define a bond. Due to the large system size, a single frame is sufficient for the radial distribution function analysis. The diffraction scattering intensity, $I(s)$, in the polycrystalline approximation was computed using the Debye scattering equation [68]

$$
I(s)=\sum_{i=1}^{N} \sum_{j=1}^{N} f_{i}(s) f_{j}(s) \frac{\sin \left(2 \pi s r_{i j}\right)}{2 \pi s r_{i j}}
$$

where $s$ is the modulus of the scattering vector, $r_{i j}$ is the distance between atoms $i$ and $j, N$ is the number of atoms and $f(s)$ is the scattering factor for a single carbon atom. The minimum image convention causes problems when using the Debye equation, and therefore it is necessary to map the atoms into the primitive cell and treat the structure as an isolated cluster. 


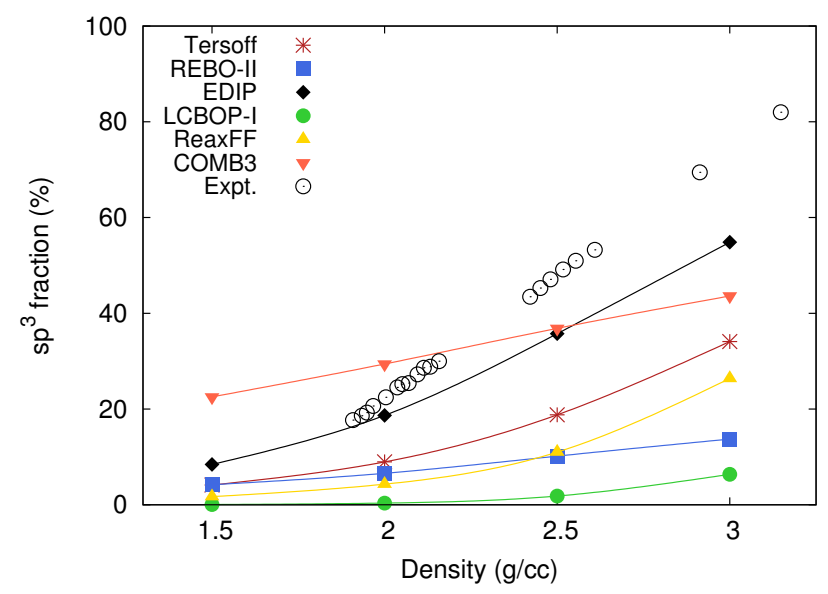

Figure 2: (Color online) Percentage fraction of $s p^{3}$ bonding as a function of density in amorphous carbons created by liquid quenching. Experimental data from Schwan et al.[69] is represented with open circles.
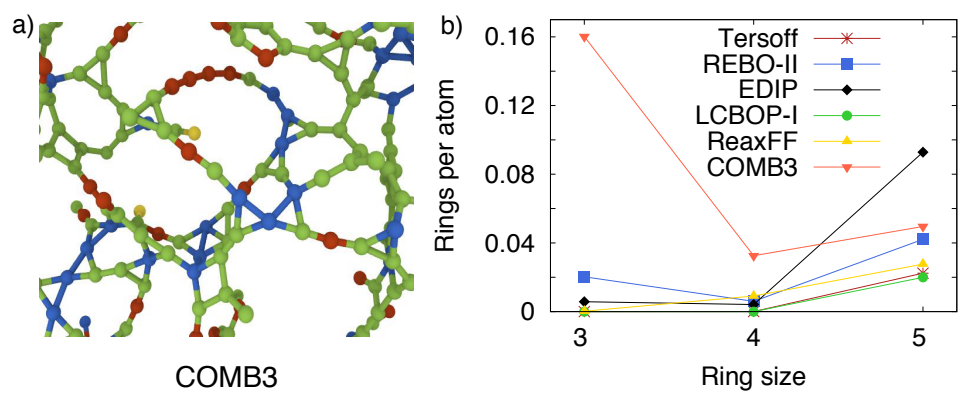

Figure 3: (Color online) (a) Close-up view of the $1.5 \mathrm{~g} / \mathrm{cc}$ amorphous structure generated using the COMB3 potential. Red, green and blue atoms denote $s p, s p^{2}$ and $s p^{3}$ bonding, respectively. Yellow atoms have just one neighbour. (b) Ring statistics up to pentagons for $1.5 \mathrm{~g} / \mathrm{cc}$ amorphous carbon using all six potentials.

\section{Results}

\subsection{Amorphous carbons}

It is well-known experimentally [69] and from ab initio simulations [70] that the $s p^{3}$ fraction of amorphous carbon varies linearly with density across a broad range. Figure 2 compares experimental values from the literature against the six potentials examined in this study. The amorphous carbon structures were generated via liquid quenching as described in the Methodology. At low densities most of the potentials predict similar $s p^{3}$ fractions, but as the density increases the values diverge significantly from each other. At the highest density of $3 \mathrm{~g} / \mathrm{cc}$ the lowest $s p^{3}$ fraction is $6.3 \%$ (LCBOP-I) while the highest is $54.9 \%$ (EDIP); for comparison the experimental value is higher still at $\sim 75 \%$. A complete list of $s p, s p^{2}$ and $s p^{3}$ fractions for the four densities and six potentials is given in Table 3 .

The COMB3 potential shows a distinctly different behavior to the other potentials, with a very high $s p^{3}$ fraction of $22.5 \%$ at low density. The origin of this behaviour became clear upon visual examination. The cross-sectional slice of a $1.5 \mathrm{~g} / \mathrm{cc}$ COMB3 amorphous carbon in Figure 3(a) reveals a large number of triangles. Almost every atom which is $s p^{3}$ bonded (denoted in blue), is part of one of these triangles. Panel (b) quantifies the number of triangles, quadrilaterals and pentagons for all six potentials, demonstrating that COMB3 predicts many more triangles than the other five potentials and to a lesser extent, quadrilaterals. This behaviour occurs across all four densities with COMB3, demonstrating that the potential has a bias towards triangles. This aspect of the potential is unphysical as ab initio calculations have shown that 

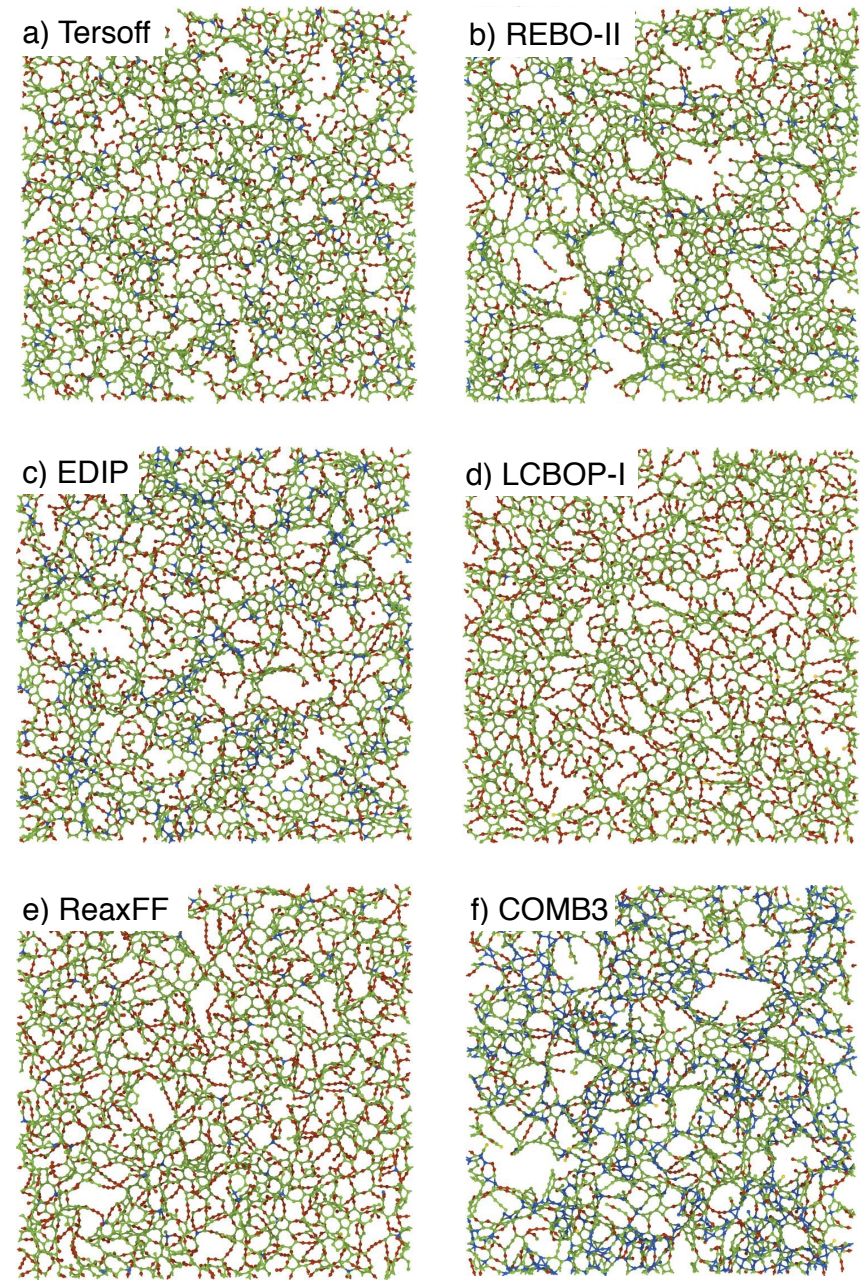

Figure 4: (Color online) Cross-sectional slice of $1 \mathrm{~nm}$ thickness for $1.5 \mathrm{~g} / \mathrm{cc}$ amorphous carbons generated using all six potentials. Red, green and blue atoms denote $s p, s p^{2}$ and $s p^{3}$ bonding, respectively.

triangular structures in amorphous carbon are typically present at very low levels of approximately 0.03 triangles per atom [71,67].

Figure 4 shows cross-sectional slices of $1.5 \mathrm{~g} / \mathrm{cc}$ amorphous carbon generated using the six potentials. Conventional wisdom holds that at low densities most carbon potentials produce similar structures and broadly speaking this is true for the potentials here with the exception of COMB3. However, closer examination reveals some small differences, in particular the presence of voids in the REBO-II structure which is not seen with the other potentials. The $s p^{2}$ fractions are around $60 \%$ for EDIP, LCBOP-I and ReaxFF, and around $75 \%$ for Tersoff and REBO-II (see Table 3). The largest differences are seen in the $s p$ fractions, where REBO-II has half the $s p$ fraction of LCBOP-I and ReaxFF. For the latter two potentials this higher $s p$ fraction is associated with long $s p$-bonded chains which are visible in Figure 4. At higher densities all the structures are fully disordered and the only visual difference is achieved when colored by the coordination number.

\subsection{Annealing}

To test the ability of the potentials to describe making and breaking of bonds, we performed annealing experiments near the melting point for each potential. As described in the Methodology, the temperature of the annealing is specific to each potential and density range, and was chosen such that there is sufficient 

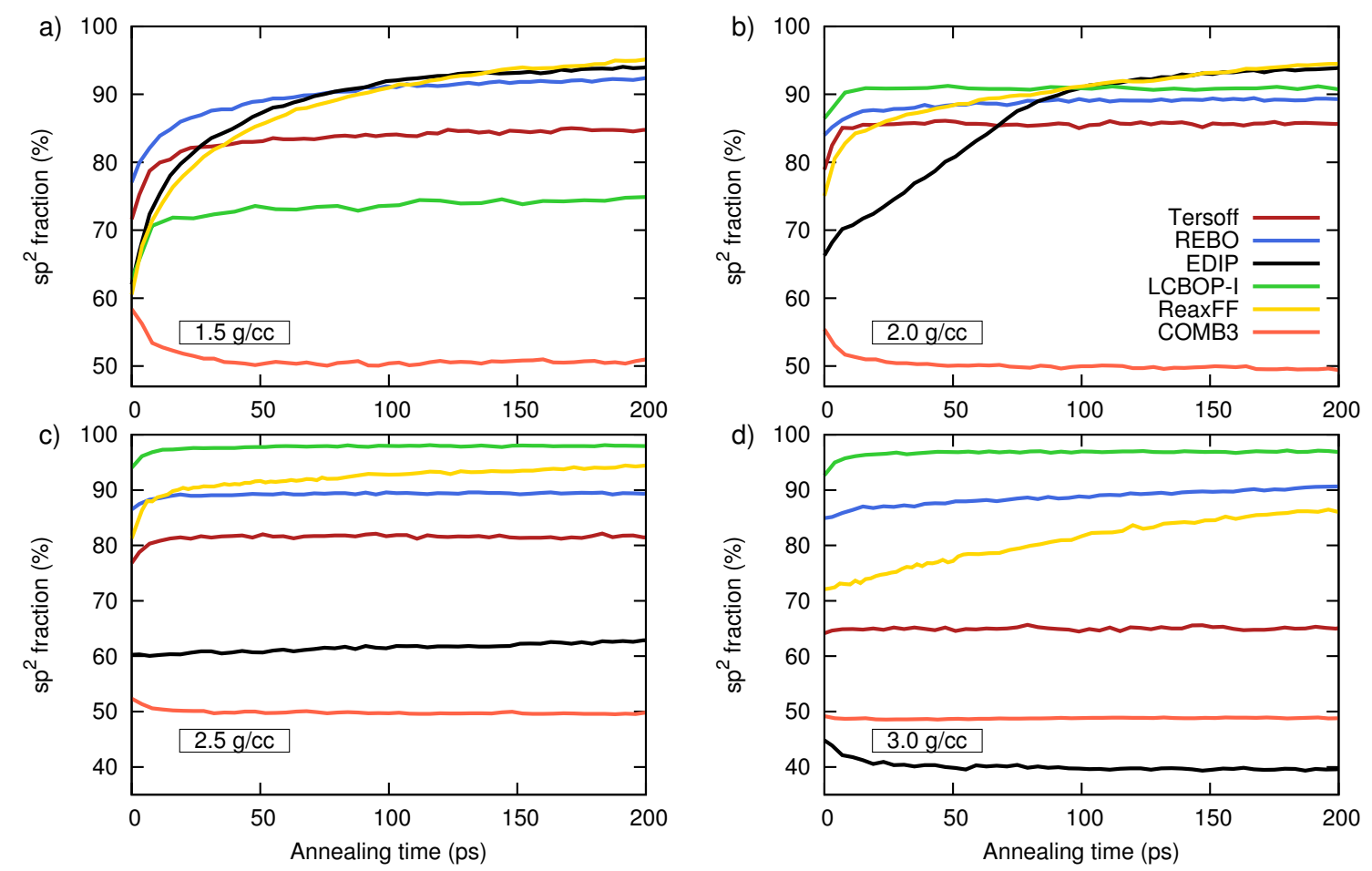

Figure 5: (Color online) Evolution of $s p^{2}$ bonding for the first $200 \mathrm{ps}$ of annealing of amorphous carbon. Data is shown for densities of (a) $1.5 \mathrm{~g} / \mathrm{cc}$, (b) $2.0 \mathrm{~g} / \mathrm{cc}$, (c) $2.5 \mathrm{~g} / \mathrm{cc}$ and (d) $3.0 \mathrm{~g} / \mathrm{cc}$ for all six potentials.

temperature to enable structural evolution but not so much that the liquid phase is reached. Using this approach a tendency towards graphite-like structures is expected at densities below that of crystalline graphite, $2.27 \mathrm{~g} / \mathrm{cc}$, while at higher densities a more $s p^{3}$-bonded structure should emerge.

Figure 5 shows the evolution of the $s p^{2}$ fraction for the first $200 \mathrm{ps}$ of annealing for each potential and density. Although several of the calculations had already reached equilibrium by this time, some systems, such as ReaxFF, required further annealing. With the exception of COMB3, all of the calculations were run for a further 200 ps. Full data on the coordination fractions after 200 and 400 ps of annealing is given in Table 3. At low densities (1.5 and $2.0 \mathrm{~g} / \mathrm{cc}$ ) most of the potentials exhibit the expected behaviour in which the $s p^{2}$ fraction increases significantly over time; the exception is COMB3 where the $s p^{2}$ fraction is reduced upon annealing. This unphysical behaviour of COMB3 occurs for all four densities, and hence COMB3 will not be included in the following discussion.

Even though most of the potentials predict increasing graphitization at low densities, there is considerable variation at the lowest density of $1.5 \mathrm{~g} / \mathrm{cc}$. The final $s p^{2}$ fraction after $400 \mathrm{ps}$ of annealing varies from as little as $76 \%$ with LCBOP-I to as high as $97 \%$ with ReaxFF. At $2.0 \mathrm{~g} / \mathrm{cc}$ the $s p^{2}$ fractions are more tightly clustered, spanning a range between 86 and $97 \%$. As the density increases the disparity between the potentials increases substantially. At $2.5 \mathrm{~g} / \mathrm{cc}$ the LCBOP-I and ReaxFF structures are almost entirely $s p^{2}$-bonded $(>95 \%)$, while the EDIP structure is only $65 \% s p^{2}$. Tersoff and REBO-II lie intermediate with around 80 and $90 \% s p^{2}$, respectively. At $3.0 \mathrm{~g} / \mathrm{cc}$ the spread is even larger, with REBO-II, LCBOP-I and ReaxFF all exhibiting $s p^{2}$ fractions above $90 \%$, Tersoff around $65 \%$ and EDIP at 39\%. At this density, which is $32 \%$ higher than that of graphite, and only $15 \%$ below that of diamond, the observation of $s p^{2}$ fractions above $90 \%$ is unrealistic.

Cross-sectional snapshots of the final annealed structures for the lowest and the highest densities are shown in Figure 6. One of the virtues of using a large simulation cell of 32,768 atoms is that the slices shown here are representative of the full network. When we varied the location or orientation of the slice the overall 

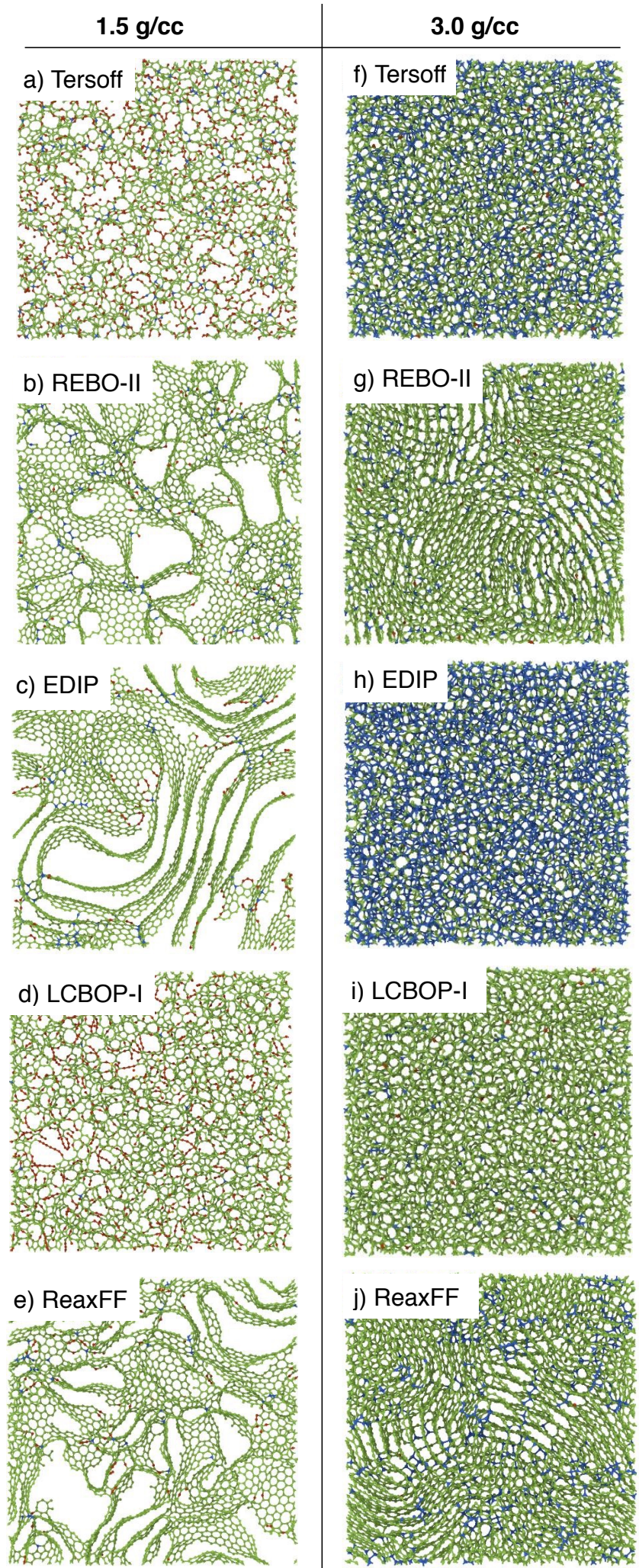

Figure 6: (Color online) Cross-sectional slice of $1 \mathrm{~nm}$ thickness for 1.5 and $3.0 \mathrm{~g} / \mathrm{cc}$ annealed carbons generated using the five potentials. Red, green and blue atoms denote $s p, s p^{2}$ and $s p^{3}$ bonding, respectively. 
trends remained unchanged. The low-density structures in Figure 6 display great variation, ranging from almost amorphous in the case of LCBOP-I to strong medium-range order with multiple graphitic layers in EDIP. With the exception of LCBOP-I, hexagons are apparent in all the structures, and assemble to form graphene fragments of varying size. Associated with this graphitization is the presence of small voids and pores which arise because of the more efficient atomic packing relative to a disordered structure. Unique to LCBOP-I are large numbers of long $s p$-bonded chains which can be seen in red in Figure 6 . All of the potentials have a significant number of $s p$ hybridized atoms in the amorphous structure but it is only with LCBOP-I that these persist to any significant degree after annealing.

The high-density structures in Figure 6 can be grouped into two categories, with Tersoff, EDIP and LCBOP-I maintaining an amorphous structure, while REBO-II and ReaxFF form highly ordered graphitic nanoclusters. As can be seen visually from the mixture of green and blue, there are significant differences in the $s p^{3}$ fraction of the amorphous structures: LCBOP-I has an $s p^{3}$ fraction of just $10 \%$, Tersoff has $34 \%$ while EDIP has $60 \%$. Visually, the REBO-II and ReaxFF structures appear similar, and can only be distinguished by further quantitative analysis.

Figure 7 shows the radial distribution function, $\mathrm{g}(\mathrm{r})$, for all six potentials and all four densities. The vertical dotted lines indicate the $\mathrm{C}-\mathrm{C}$ bond length for graphite $(1.421 \AA)$ and diamond $(1.547 \AA)$. The data again confirms that there are significant differences between the potentials at all densities. At 1.5 g/cc, EDIP, REBO-II and ReaxFF show a narrow and sharp first neighbor peak at the graphite distance, corresponding to their large $s p^{2}$ fractions and high degree of graphitization. A satellite first neighbor peak is clearly distinct for LCBOP-I at very short distances, $1.33 \AA$; this corresponds to the $s p$ chains described above. For the Tersoff potential the first neighbor peak is situated intermediate between graphite and diamond and it is significantly broader, indicating a lower degree of crystallinity. Although slightly obscured, the main first neighbor peak of LCBOP-I is similarly broad, indicative of its amorphous character. While less straightforward to interpret, the second neighbor peak also shows large variation between potentials.

At $2.0 \mathrm{~g} / \mathrm{cc}$ the radial distribution function, Figure $7 \mathrm{~b}$, is broadly similar to that at $1.5 \mathrm{~g} / \mathrm{cc}$. The main differences are the absence of the $s p$-bonded peak with LCBOP-I and the reduced height of the first neighbor peak for all the potentials. The reduction in the peak height reflects the more compact nature of the $2.0 \mathrm{~g} / \mathrm{cc}$ structures, in which voids are largely absent and the motion of atoms during annealing is more restricted. At $2.5 \mathrm{~g} / \mathrm{cc}$ the radial distribution function, Figure 7c, can be grouped into two families: REBOII, LCBOP-I and ReaxFF all have relatively sharp peaks at the graphite bondlength, while Tersoff and EDIP have much broader peaks and at a larger distance. The REBO-II structure also exhibits a shoulder in the radial distribution function at the diamond bondlength, reflecting a modest amount of $s p^{3}$ bonding (8.6\%). As seen in Table 3, at this density ReaxFF and LCBOP-I have extremely low $s p^{3}$ fractions, while Tersoff and EDIP are much higher, at 16 and $34 \%$ respectively. At the highest density of $3.0 \mathrm{~g} / \mathrm{cc}$ these two groupings are maintained, with the main change being that the first neighbour peak of EDIP shifts towards diamond, consistent with the high $s p^{3}$ fraction of $61 \%$. EDIP is the only potential where higher density correlates with a shift of the first neighbour peak towards diamond. Surprisingly, LCBOP-I exhibits the opposite behavior, where the main peak shifts to shorter distances as the density increases. For the other potentials, little change with density occurs. It is important to note that the groupings based on g(r) differ from groupings based on visual examination of the structures. In the visual examination, LCBOP-I was grouped as amorphous with EDIP and Tersoff, while in the $\mathrm{g}(\mathrm{r})$ analysis it is grouped with REBO-II and ReaxFF based on the graphitic first-neighbor peak.

Ring statistics provide a measure of the topology and are a useful way to distinguish between structures which may otherwise appear similar in radial distribution function analysis. Figure 8 shows the number of rings per atom for all potentials and all four densities. As a reference point, there are 0.5 hexagons per atom in graphite and 2 hexagons per atom in diamond. With the exception of EDIP at $3.0 \mathrm{~g} / \mathrm{cc}$, all of the structures have fewer hexagons than ideal graphite. Once again, there are large difference between the potentials across all densities and visually, the differences are even larger than those seen for $\mathrm{g}(\mathrm{r})$.

At the lowest density of $1.5 \mathrm{~g} / \mathrm{cc}$, EDIP, REBO-II and ReaxFF produce the largest number of hexagons, consistent with the images in Figure 6. For these potentials the number of pentagons and heptagons is similar, typical of defective graphitic sheets. A flat ring distribution is seen with LCBOP-I, indicative of a lack of crystallinity. The ring distribution for Tersoff is mostly flat with a small peak of hexagons, and so 

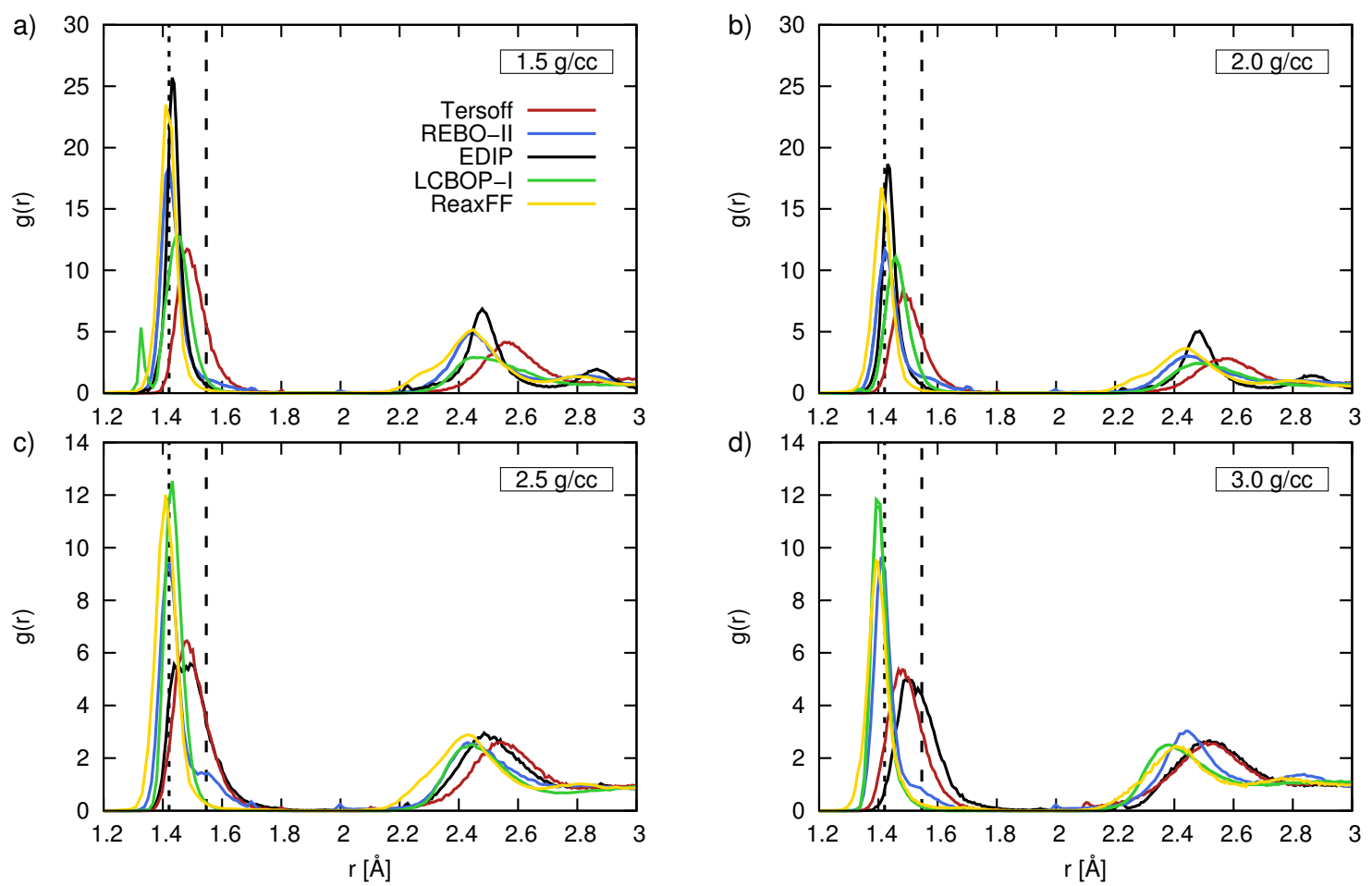

Figure 7: (Color online) Radial distribution function of the annealed structures. Data is shown for densities of (a) $1.5 \mathrm{~g} / \mathrm{cc}$, (b) $2.0 \mathrm{~g} / \mathrm{cc}$, (c) $2.5 \mathrm{~g} / \mathrm{cc}$ and (d) $3.0 \mathrm{~g} / \mathrm{cc}$. The vertical dotted lines indicate the bond length for graphite and diamond.

lies intermediate between these two extremes. The ring distribution at $2.0 \mathrm{~g} / \mathrm{cc}$ follows the same trends as at $1.5 \mathrm{~g} / \mathrm{cc}$, except that REBO-II and Tersoff contain fewer hexagons. At $2.5 \mathrm{~g} / \mathrm{cc}$, only EDIP and ReaxFF show any significant preference for hexagons, and both potentials show few rings larger than octagons. In contrast, REBO-II, Tersoff and LCBOP-I all have a substantial number of large rings. We note that despite showing similar $\mathrm{g}(\mathrm{r})$ data in Figure $7(\mathrm{c})$, the corresponding ring statistics for Tersoff and EDIP are significantly different. At $3.0 \mathrm{~g} / \mathrm{cc}$, ReaxFF and REBO-II show the characteristic ring distribution for a graphite-like structure with defects. While this is consistent with the layered regions seen in Figure 6, it is against experimental observations, where a density of $3.0 \mathrm{~g} / \mathrm{cc}$ is incompatible with graphitic ordering. The results at $3.0 \mathrm{~g} / \mathrm{cc}$ for Tersoff and LCBOP-I are similar to those at $2.5 \mathrm{~g} / \mathrm{cc}$. At this high density, EDIP is quite different to the other potentials due to the large number of $s p^{3}$ bonds, which increases the number of hexagons above the graphite value of 0.5 hexagons per atom. At this density, EDIP also has a significant number of large rings, indicative of an amorphous structure.

The diffraction intensity computed using the Debye scattering equation provides an elegant means to quantify the degree of 002 ordering between graphitic layers. Figure 9 shows the scattering intensity for all potentials and densities, with the vertical dashed line at $s=1 / 3.35 \AA^{-1}$ indicating the position of the 002 peak for ideal graphite. At $1.5 \mathrm{~g} / \mathrm{cc}$ the only potential to exhibit any significant 002 ordering is EDIP, consistent with the image in Figure 6(c). However, this peak occurs at a smaller value of $s$ as compared to graphite, meaning that the layers are more widely spaced. Several of the potentials (EDIP, ReaxFF and REBO-II) have a peak at $s=0.48 \AA^{-1}$, corresponding to 100 and 101 planes in graphite with a mean spacing of $2.08 \AA$. At large values of $s$, there is a small peak at $0.82 \AA^{-1}$ (distance $=1.22 \AA$ ) corresponding to the graphitic 110 reflection. At $2.0 \mathrm{~g} / \mathrm{cc}$ both ReaxFF and EDIP have a sharp 002 peak close to the graphite value, while LCBOP-I and Tersoff have broad peaks. However, neither LCBOP-I nor Tersoff show a 100/101 peak at $0.48 \AA^{-1}$, while ReaxFF and EDIP do. These signatures of graphitization for ReaxFF and EDIP are consistent with the ring statistics which show many hexagons, a few pentagons and heptagons, and almost 

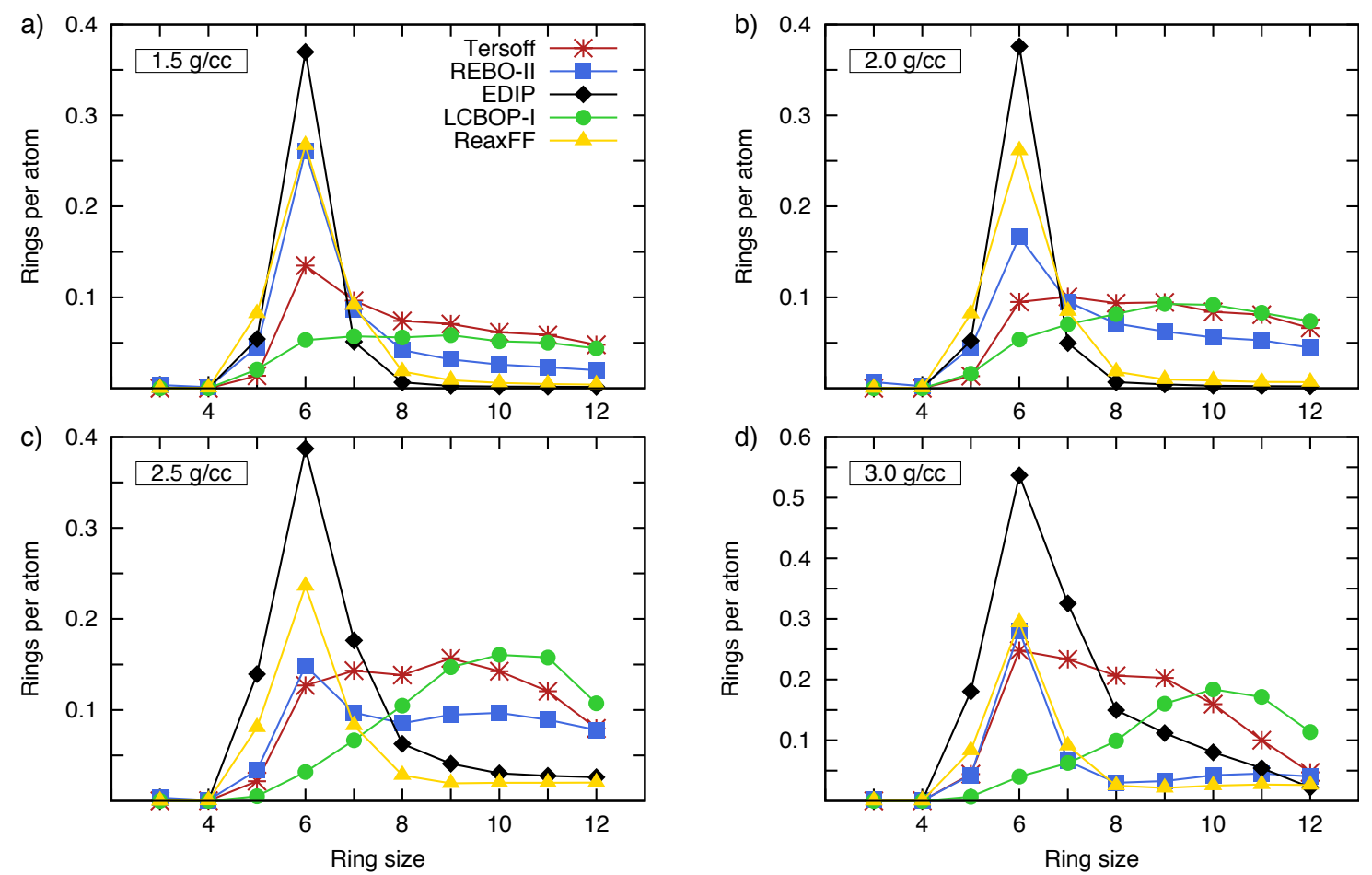

Figure 8: (Color online) Number of rings per atom for polygons from triangles to dodecagons for the annealed structures. Data is shown for densities of (a) $1.5 \mathrm{~g} / \mathrm{cc}$, (b) $2.0 \mathrm{~g} / \mathrm{cc}$, (c) $2.5 \mathrm{~g} / \mathrm{cc}$ and (d) $3.0 \mathrm{~g} / \mathrm{cc}$. 

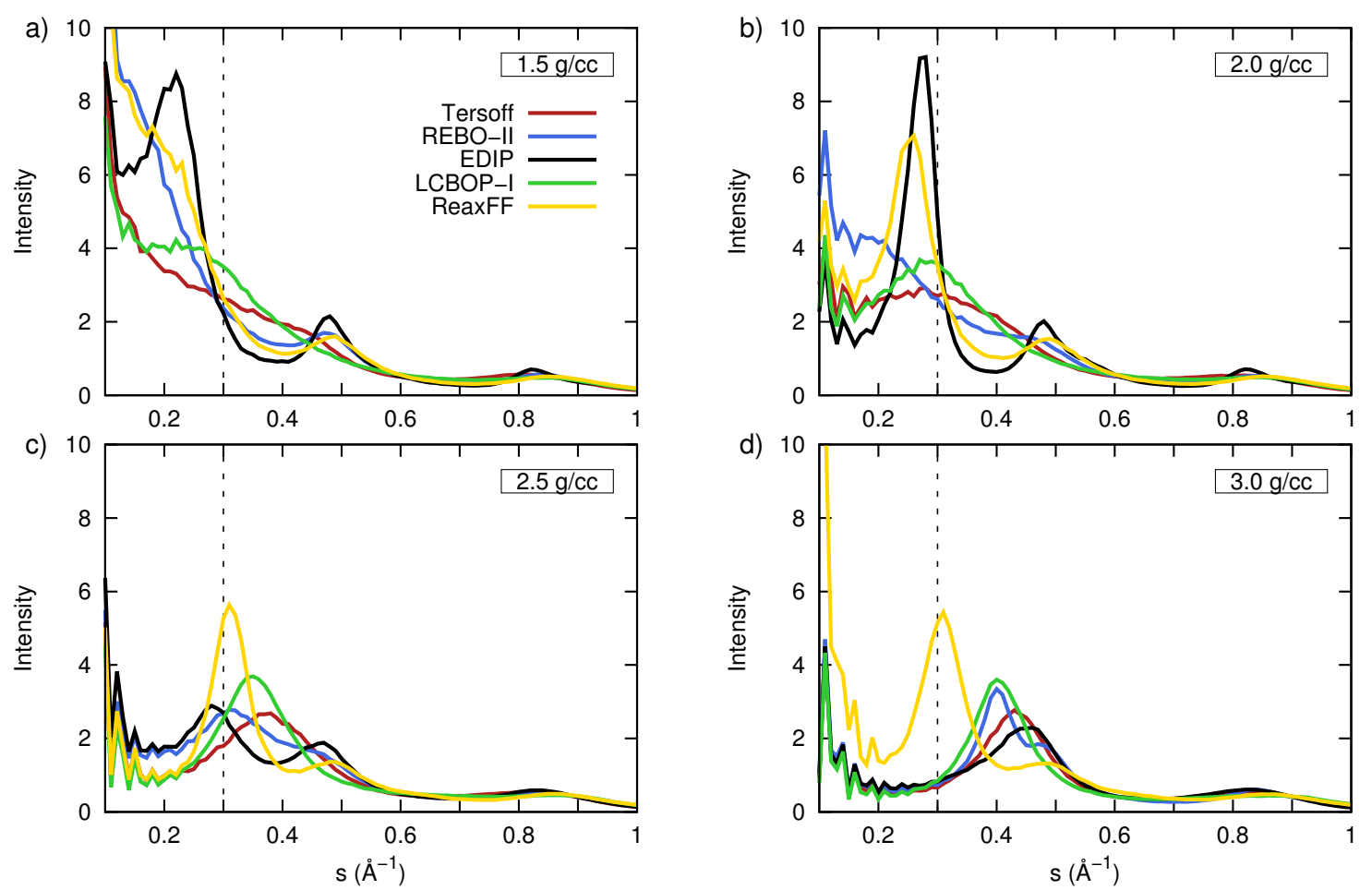

Figure 9: (Color online) Diffraction intensity computed using the Debye scattering equation for the annealed structures. Data is shown for densities of (a) $1.5 \mathrm{~g} / \mathrm{cc}$, (b) $2.0 \mathrm{~g} / \mathrm{cc}$, (c) $2.5 \mathrm{~g} / \mathrm{cc}$ and (d) $3.0 \mathrm{~g} / \mathrm{cc}$. The dashed line indicates the position of the 002 scattering peak corresponding to the graphite interlayer spacing of $3.35 \AA$.

no large polygons. At this density of $2.0 \mathrm{~g} / \mathrm{cc}, \mathrm{REBO}-\mathrm{II}$ exhibits a shoulder at $0.48 \AA^{-1}$, consistent with the reduction in the number of hexagons as compared to $1.5 \mathrm{~g} / \mathrm{cc}$.

At $2.5 \mathrm{~g} / \mathrm{cc}$ ReaxFF shows a sharp 002 peak, while a smaller 002 peak can be seen for EDIP. Both ReaxFF and EDIP still show a second peak at $0.48 \AA^{-1}$ which can be associated with the 100 and 101 planes. The other three potentials show one single broad peak, indicating a lack of crystallinity. At $3.0 \mathrm{~g} / \mathrm{cc}$ ReaxFF continues to show a sharp 002 peak and a smaller 100/101 peak. The same peaks are also present for REBO-II, however the 002 peak is displaced to higher $s$, corresponding to smaller interlayer distances of around $2.5 \AA$. Consistent with the visual examination, the other potentials are amorphous and show a single broad peak.

\section{Discussion}

The analysis performed in this work shows that the properties of amorphous and annealed carbons are highly sensitive to the choice of potential. In the case of amorphous carbons, the predicted $s p^{3}$ fraction at $3.0 \mathrm{~g} / \mathrm{cc}$ is uniformly spread from as little as $6 \%$ to as high as $55 \%$. For less dense structures the potentials produce more similar results, however, the best performing potential at all densities is EDIP. This observation is not especially surprising as EDIP was developed with amorphous carbon film growth in mind. The worst-performing potential is COMB3, which has an unrealistically high abundance of triangles. One possible explanation is that the bond-angle term is too soft and needs to be reparametrized.

While liquid quenching has previously been used to compare the performance of carbon potentials [67, 72], the use of annealing to study transferability is new. We were surprised by the variety of structures produced at both high and low densities. Once again, COMB3 performed poorly during annealing, with the incorrect result of decreasing $s p^{2}$ fraction for all densities. In its current form, COMB3 appears unsuitable for all types 
of solid carbon. For the other potentials it is less straightforward to rank their performance. For amorphous carbons there is experimental and ab initio data to compare against, but there is no direct experimental equivalent of the annealing processes examined here and the system size precludes ab initio study. That said, there is lot of knowledge in the community regarding the effects of heat on low-density carbon materials. Pyrolysis of carbonaceous materials produces carbonized solids with a layered microstructure and high $s p^{2}$ fractions. These materials are of economic importance and include glassy carbon and activated carbons. The two low densities studied here are typical for glassy carbon. It is less obvious how to quantify the expected behavior of the annealing simulations at high density. Tetrahedral amorphous carbon, which has a density around $3 \mathrm{~g} / \mathrm{cc}$, is known to retain its structure at temperatures as high as $1400 \mathrm{~K}$ [73], while high-pressure/high-temperature experiments show that carbon favors structures with a high $s p^{3}$ fraction $[74,75,76]$. Our calculations are performed at constant volume, and since the application of high temperature imposes a pressure, we anticipate that the high density simulations should also generate high $s p^{3}$ fractions.

The Tersoff potential produces disordered structures at all densities, and does not show graphitization under annealing. This behavior occurs due to the absence of a dihedral (also known as torsion) term in the potential to penalise rotation of $\pi$-bonds. Since this penalty is not present, ethylene-like units can freely rotate about the axis of the $s p^{2}$ bond, and entropy will not favor planar arrangements. With the exception of EDIP, Tersoff provides the best reproduction of a high $s p^{3}$ fraction at high density. Although not studied here, this behavior can be improved further by increasing the cutoff from $2.1 \AA$ to $\sim 2.45 \AA$, slightly below the second-neighbour bondlength of graphite $[16,8,19]$.

At low densities, REBO-II shows short-range order but no stacked layers. Individual graphitic fragments are present as REBO-II includes a dihedral absent in Tersoff. Since REBO-II lacks the long-range of AIREBO, this result could be improved upon if the latter was available in LAMMPS. At 3.0 g/cc REBO-II produces unreasonable results, with an 002-like peak at $s=0.4 \AA^{-1}$, corresponding to an interlayer distance of $2.5 \AA$. If graphite were compressed from its normal spacing of $3.35 \AA$ down to $2.5 \AA$, this unrealistic graphite would have a density of $2.27 \times 3.35 / 2.5=3.04 \mathrm{~g} / \mathrm{cc}$, exactly the same as the calculation.

EDIP produces stacked layered structures at the lowest densities, and is the only potential to exhibit stacking at $1.5 \mathrm{~g} / \mathrm{cc}$. This stacking behavior could seem surprising, as EDIP does not include van der Waals attraction. However, EDIP does include long-range $\pi$-repulsion out to $3.2 \AA$, and this is sufficient to drive ordering behavior, as seen in previous simulations of double-walled nanotubes derived from carbon peapods [56]. While the 002 peak is at the correct location at $2.0 \mathrm{~g} / \mathrm{cc}$, it shifts to $s=0.22 \AA^{-1}$ (i.e. a spacing of $4.5 \AA$ ) at $1.5 \mathrm{~g} / \mathrm{cc}$ as in the absence of attraction the only constraint is the volume. At $3.0 \mathrm{~g} / \mathrm{cc}$, EDIP predicts an amorphous structure, and is the only potential to predict a high $s p^{3}$ fraction.

All of the ReaxFF structures exhibit high $s p^{2}$ fractions and few large polygons, indicative of a a high degree of short-range-ordering. Stacked layers are evident at all densities except $1.5 \mathrm{~g} / \mathrm{cc}$. Due to the presence of long-range attraction, the 002 peak is located at the expected position corresponding to an interlayer distance of $3.35 \AA$. However, ReaxFF appears to have a bias towards $s p^{2}$ bonding, as even at the relatively high density of $3.0 \mathrm{~g} / \mathrm{cc}$ the $s p^{3}$ fraction is less than $10 \%$. Similar to REBO-II, the observation of graphitic-like sheets at this density is unrealistic.

The LCBOP-I potential performs poorly across all densities. The low density structures do not graphitize, and the high density structures do not have high $s p^{3}$ fractions. The LCBOP-I structures are all amorphous, and hexagons are not favored. As the density increases, the number of large polygons far exceeds the number of hexagons, indicating that the structure has neither graphitic nor diamond-like elements. It would be instructive to repeat the simulations here using LCBOP-II to evaluate whether the better description of bond dissociation improves performance.

In summary, none of the carbon potentials here can be considered to provide a universal description of solid carbon. EDIP performs well overall, but the absence of long-range attraction means the interlayer spacing increases without bound at very low densities. ReaxFF performs well in the low-density range, but it does not perform well at high density in either the amorphous or annealing simulations. The absence of a dihedral term in the Tersoff potential makes it generally unsuitable to study graphitization, but it does provide a reasonable description of amorphous carbon at low computational cost. REBO-II exhibits unrealistic behaviour at high densities and has a preference for $s p^{2}$ bonding, a bias it shares with ReaxFF. LCBOP-I does not perform well at any density, and is not suitable to study either graphitization or amorphous carbon. 
These observations show the need to continue developing carbon potentials and to perform systematic testing. Given its predominance in the computational community, LAMMPS is well-suited for this task. Ideally, all potentials in LAMMPS should be evaluated in a similar manner as tested here. Without such a test, the transferability is difficult to assess. Furthermore, existing potentials such as LCBOP-II need to be coded into LAMMPS, as should be any future potentials. Finally, the analysis of the different potentials provides guidelines towards the characteristics of an ultimate potential for carbon. Such an ideal potential should include a full bond-order treatment, dihedral terms to promote planarity, long-range attraction to capture van der Waals, and a carefully chosen cutoff/screening term to describe bond-making and breaking. Additionally, the potential should be extensible to other elements, especially hydrogen and oxygen.

\section{Conclusion}

In this work we have compared the performance of six carbon potentials and evaluated their ability to describe amorphous carbons and the graphitization process. At low densities EDIP and ReaxFF provide the most accurate picture, while at high density, only EDIP is able to predict high $s p^{3}$ fractions and correct microstructure. From a general perspective, the main observation is the enormous variation between the different potentials. Indeed, it is disconcerting that no two potentials give the same results, either for the amorphous or annealed structures. This raises the question when to use each potential. Ultimately, no potential is perfect, and it is the responsibility of the researcher to thoroughly evaluate the transferability for each particular case. Finally, we hope that this article serves as an impetus for developers of interatomic carbon potentials to make their routines available in LAMMPS and to test them against challenging targets.

Acknowledgments This work was supported by Australian Research Council Discovery Project DP150103487. NAM acknowledges the fellowship FT120100924. ISM acknowledges the fellowship FT140100191. Computational resources are provided by the Pawsey Supercomputing Centre with funding from the Australian Government and the Government of Western Australia.

\section{Appendix I}

Tables 4 and 5 show cohesive energy, lattice constant, bond length and density for graphite and diamond crystals respectively. Values are computed with the potentials as implemented in LAMMPS and experimental values are shown for comparison.

\section{Appendix II}

Computational cost for each potential is described in Table 6 for the case of annealing of the amorphous carbons with density $1.5 \mathrm{~g} / \mathrm{cc}$ and $3.0 \mathrm{~g} / \mathrm{cc}$. Processor times are expressed in seconds per-atom per-timestep for a single CPU. For ReaxFF and COMB3 the charge equilibration calculation has been switched off. ReaxFF is roughly 5 times more expensive than EDIP.

Table 4: Graphite properties. Graphene cohesive energy is indicated in parentheses as a reference. Experimental values are from Ref. [77].

\begin{tabular}{lcccc} 
Potential & $\begin{array}{c}\mathrm{E}_{\text {coh }} \\
(\mathrm{eV} / \text { atom })\end{array}$ & $\begin{array}{c}\text { c-Spacing } \\
(\AA)\end{array}$ & $\begin{array}{c}\text { Bond length } \\
(\AA)\end{array}$ & $\begin{array}{c}\text { Density } \\
(\mathrm{g} / \mathrm{cc})\end{array}$ \\
\hline Tersoff & $7.393(7.393)$ & 2.100 & 1.461 & 3.427 \\
\hline REBO-II & $7.807(7.807)$ & 2.001 & 1.398 & 3.741 \\
\hline EDIP & $7.363(7.363)$ & 3.201 & 1.420 & 2.379 \\
\hline LCBOP-I & $7.375(7.351)$ & 3.349 & 1.419 & 2.276 \\
\hline ReaxFF & $7.594(7.525)$ & 3.350 & 1.420 & 2.272 \\
\hline COMB3 & $7.403(7.352)$ & 3.351 & 1.420 & 2.271 \\
\hline Experiment & $7.374(-)$ & 3.355 & 1.421 & 2.267
\end{tabular}


Table 5: Diamond properties. Experimental values from Ref. [77]

\begin{tabular}{lcccc} 
Potential & $\begin{array}{c}\mathrm{E}_{\text {coh }} \\
(\mathrm{eV} / \text { atom })\end{array}$ & $\begin{array}{c}\text { Lattice constant } \\
(\AA)\end{array}$ & $\begin{array}{c}\text { Bond length } \\
(\AA)\end{array}$ & $\begin{array}{c}\text { Density } \\
(\mathrm{g} / \mathrm{cc})\end{array}$ \\
\hline Tersoff & 7.368 & 3.566 & 1.544 & 3.519 \\
\hline REBO-II & 7.370 & 3.566 & 1.544 & 3.519 \\
\hline EDIP & 7.347 & 3.573 & 1.547 & 3.499 \\
\hline LCBOP-I & 7.351 & 3.565 & 1.544 & 3.521 \\
\hline ReaxFF & 7.567 & 3.578 & 1.549 & 3.484 \\
\hline COMB3 & 7.330 & 3.547 & 1.536 & 3.576 \\
\hline Experiment & 7.349 & 3.567 & 1.545 & 3.516
\end{tabular}

Table 6: Computational cost per CPU for each potential during the annealing of the amorphous carbons.

\begin{tabular}{lrr} 
Potential & \multicolumn{1}{c}{ Cost $(\mu \mathrm{s} /$ atom/timestep $)$} \\
\cline { 2 - 3 } & $1.5 \mathrm{~g} / \mathrm{cc}$ & $3.0 \mathrm{~g} / \mathrm{cc}$ \\
\hline Tersoff & 3.2 & 4.8 \\
\hline REBO-II & 5.3 & 7.2 \\
\hline EDIP & 62.4 & 195.3 \\
\hline ReaxFF & 345.9 & 885.5 \\
\hline LCBOP-I & 9.9 & 16.9 \\
\hline COMB3 & 158.7 & 304.3
\end{tabular}

\section{References}

[1] J. B. Gibson, A. N. Goland, M. Milgram, G. H. Vineyard, Dynamics of radiation damage, Phys. Rev. 120 (4) (1960) 1229-1253. doi:10.1103/PhysRev.120.1229.

[2] M. S. Daw, M. I. Baskes, Embedded-atom method: Derivation and application to impurities, surfaces, and other defects in metals, Phys. Rev. B 29 (12) (1984) 6443-6453. doi:10.1103/PhysRevB.29.6443.

[3] M. W. Finnis, J. E. Sinclair, A simple empirical N-body potential for transition metals, Philos. Mag. A 50 (1) (1984) 45-55. doi:10.1080/01418618408244210.

[4] J. Tersoff, New empirical model for the structural properties of silicon, Phys. Rev. Lett. 56 (6) (1986) $632-635$. doi:10.1103/PhysRevLett.56.632.

[5] J. Tersoff, Empirical interatomic potential for carbon, with applications to amorphous carbon, Phys. Rev. Lett. 61 (25) (1988) 2879-2882. doi:10.1103/PhysRevLett.61.2879.

[6] D. W. Brenner, Empirical potential for hydrocarbons for use in simulating the chemical vapor deposition of diamond films, Phys. Rev. B 42 (15) (1990) 9458-9471. doi:10.1103/PhysRevB.42.9458.

[7] J. Tersoff, Carbon defects and defect reactions in silicon, Phys. Rev. Lett. 64 (15) (1990) 1757-1760. doi:10.1103/PhysRevLett.64.1757.

[8] K. Nordlund, J. Keinonen, T. Mattila, Formation of ion irradiation induced small-scale defects on graphite surfaces, Phys. Rev. Lett. 77 (4) (1996) 699-702. doi:10.1103/PhysRevLett.77.699.

[9] S. J. Stuart, A. B. Tutein, J. A. Harrison, A reactive potential for hydrocarbons with intermolecular interactions, J. Chem. Phys. 112 (14) (2000) 6472-6486. doi:10.1063/1.481208.

[10] D. W. Brenner, O. A. Shenderova, J. A. Harrison, S. J. Stuart, B. Ni, S. B. Sinnott, A second-generation reactive empirical bond order (REBO) potential energy expression for hydrocarbons, J. Phys.: Condens. Matter 14 (4) (2002) 783-802. doi:10.1088/0953-8984/14/4/312.

[11] S. Plimpton, Fast parallel algorithms for short-range molecular dynamics, J. Chem. Phys. 117 (1) (1995) 1-19. doi:10.1006/jcph.1995.1039.

[12] I. T. Todorov, W. Smith, K. Trachenko, M. T. Dove, DL_POLY_3: new dimensions in molecular dynamics simulations via massive parallelism, J. Mater. Chem. 16 (20) (2006) 1911-1918. doi:10.1039/B517931A.

[13] J. D. Gale, GULP: A computer program for the symmetry-adapted simulation of solids, J. Chem. Soc., Faraday Trans. 93 (4) (1997) 629-637. doi:10.1039/A606455H.

[14] J. Tersoff, Modeling solid-state chemistry: Interatomic potentials for multicomponent systems, Phys. Rev. B 39 (8) (1989) 5566-5568. doi:10.1103/PhysRevB.39.5566.

[15] J. Tersoff, Chemical order in amorphous silicon carbide, Phys. Rev. B 49 (23) (1994) $16349-16352$. 
doi:10.1103/PhysRevB.49.16349.

[16] M. Tang, S. Yip, Atomistic simulation of thermomechanical properties of $\beta$-SiC, Phys. Rev. B 52 (21) (1995) $15150-15159$. doi:10.1103/PhysRevB.52.15150.

[17] F. de Brito Mota, J. F. Justo, A. Fazzio, Structural properties of amorphous silicon nitride, Phys. Rev. B 58 (13) (1998) 8323-8328. doi:10.1103/PhysRevB.58.8323.

[18] K. Matsunaga, C. Fisher, H. Matsubara, Tersoff potential parameters for simulating cubic boron carbonitrides, Jpn. J. Appl. Phys. Pt. 2, Letters 39 (1A/B) (2000) L48-L51. doi:10.1143/JJAP.39.L48.

[19] J. T. Titantah, D. Lamoen, $\mathrm{sp}^{3} / \mathrm{sp}^{2}$ characterization of carbon materials from first-principles calculations: X-ray photoelectron versus high energy electron energy-loss spectroscopy techniques, Carbon 43 (6) (2005) 1311-1316. doi:10.1016/j.carbon.2005.01.002.

[20] O. Kum, F. H. Ree, S. J. Stuart, C. J. Wu, Molecular dynamics investigation on liquid-liquid phase change in carbon with empirical bond-order potentials, J. Chem. Phys. 119 (12) (2003) 6053-6056. doi:10.1063/1.1601216.

[21] B. Ni, K.-H. Lee, S. B. Sinnott, A reactive empirical bond order (REBO) potential for hydrocarbon-oxygen interactions, J. Phys.: Condens. Matter 16 (41) (2004) 7261-7275. doi:10.1088/0953-8984/16/41/008.

[22] A. Liu, S. J. Stuart, Empirical bond-order potential for hydrocarbons: Adaptive treatment of van der Waals interactions, J. Comput. Chem. 29 (4) (2008) 601-611. doi:10.1002/jcc.20817.

[23] L. Pastewka, P. Pou, R. Pérez, P. Gumbsch, M. Moseler, Describing bond-breaking processes by reactive potentials: Importance of an environment-dependent interaction range, Phys. Rev. B 78 (16) (2008) 161402 (R). doi:10.1103/PhysRevB.78.161402.

[24] M. T. Knippenberg, P. T. Mikulski, K. E. Ryan, S. J. Stuart, G. Gao, J. A. Harrison, Bond-order potentials with split-charge equilibration: Application to C-, H-, and O-containing systems, J. Chem. Phys. 136 (16) (2012) 164701. doi:10.1063/1.4704800.

[25] R. Perriot, X. Gu, Y. Lin, V. V. Zhakhovsky, I. I. Oleynik, Screened environment-dependent reactive empirical bond-order potential for atomistic simulations of carbon materials, Phys. Rev. B 88 (6) (2013) 064101. doi:10.1103/PhysRevB.88.064101.

[26] T. C. O'Connor, J. Andzelm, M. O. Robbins, AIREBO-M: A reactive model for hydrocarbons at extreme pressures, J. Chem. Phys. 142 (2) (2015) 024903. doi:10.1063/1.4905549.

[27] M. I. Heggie, Semiclassical interatomic potential for carbon and its application to the self-interstitial in graphite, J. Phys.: Condens. Matter 3 (18) (1991) 3065-3079. doi:10.1088/0953-8984/3/18/002.

[28] E. P. Andribet, J. Domínguez-Vázquez, A. M. C. Pérez-Martín, E. V. Alonso, J. J. Jiménez-Rodríguez, Empirical approach for the interatomic potential of carbon, Nucl. Instr. Meth. Phys. Res. B 115 (1-4) (1996) 501-504. doi:10.1016/0168$583 X(96) 01527-3$.

[29] J. Q. Broughton, M. J. Mehl, Transferable potential for carbon without angular terms, Phys. Rev. B 59 (14) (1999) 9259-9270. doi:10.1103/PhysRevB.59.9259.

[30] D. G. Pettifor, I. I. Oleinik, Analytic bond-order potentials beyond Tersoff-Brenner. I. Theory, Phys. Rev. B 59 (13) (1999) 8487-8499. doi:10.1103/PhysRevB.59.8487.

[31] I. I. Oleinik, D. G. Pettifor, Analytic bond-order potentials beyond Tersoff-Brenner. II. Application to the hydrocarbons, Phys. Rev. B 59 (13) (1999) 8500-8507. doi:10.1103/PhysRevB.59.8500.

[32] M. Mrovec, M. Moseler, C. Elsässer, P. Gumbsch, Atomistic modeling of hydrocarbon systems using analytic bond-order potentials, Prog. Mater. Sci. 52 (2-3) (2007) 230-254. doi:10.1016/j.pmatsci.2006.10.012.

[33] X. W. Zhou, D. K. Ward, M. E. Foster, An analytical bond-order potential for carbon, J. Comput. Chem. 36 (23) (2015) 1719-1735. doi:10.1002/jcc.23949.

[34] N. A. Marks, Generalizing the environment-dependent interaction potential for carbon, Phys. Rev. B 63 (3) (2000) 035401. doi:10.1103/PhysRevB.63.035401.

[35] A. C. T. van Duin, S. Dasgupta, F. Lorant, W. A. Goddard III, ReaxFF: A reactive force field for hydrocarbons, J. Phys. Chem. A 105 (41) (2001) 9396-9409. doi:10.1021/jp004368u.

[36] K. Chenoweth, A. C. T. van Duin, W. A. Goddard III, ReaxFF reactive force field for molecular dynamics simulations of hydrocarbon oxidation, J. Phys. Chem. A 112 (5) (2008) 1040-1053. doi:10.1021/jp709896w.

[37] L. Liu, Y. Liu, S. V. Zybin, H. Sun, W. A. Goddard III, ReaxFF-lg: Correction of the ReaxFF reactive force field for London dispersion, with applications to the equations of state for energetic materials, J. Phys. Chem. A 115 (40) (2011) 11016-11022. doi:10.1021/jp201599t.

[38] S. G. Srinivasan, A. C. T. van Duin, P. Ganesh, Development of a ReaxFF potential for carbon condensed phases and its application to the thermal fragmentation of a large fullerene, J. Phys. Chem. A 119 (4) (2015) 571-580. doi:10.1021/jp510274e.

[39] J. H. Los, A. Fasolino, Intrinsic long-range bond-order potential for carbon: Performance in Monte Carlo simulations of graphitization, Phys. Rev. B 68 (2) (2003) 24107. doi:10.1103/PhysRevB.68.024107.

[40] L. M. Ghiringhelli, J. H. Los, E. J. Meijer, A. Fasolino, D. Frenkel, High-pressure diamondlike liquid carbon, Phys. Rev. B 69 (10) (2004) 100101(R). doi:10.1103/PhysRevB.69.100101.

[41] J. H. Los, L. M. Ghiringhelli, E. J. Meijer, A. Fasolino, Improved long-range reactive bond-order potential for carbon. I. Construction, Phys. Rev. B 72 (21) (2005) 214102-14. doi:10.1103/PhysRevB.72.214102.

[42] L. M. Ghiringhelli, J. H. Los, A. Fasolino, E. J. Meijer, Improved long-range reactive bond-order potential for carbon. II. Molecular simulation of liquid carbon, Phys. Rev. B 72 (21) (2005) 214103. doi:10.1103/PhysRevB.72.214103.

[43] P. Erhart, K. Albe, Analytical potential for atomistic simulations of silicon, carbon, and silicon carbide, Phys. Rev. B 71 (3) (2005) 35211. doi:10.1103/PhysRevB.71.035211.

[44] B.-J. Lee, J. W. Lee, A modified embedded atom method interatomic potential for carbon, Calphad 29 (1) (2005) 7-16. doi:10.1016/j.calphad.2005.02.003. 
[45] J. Uddin, M. I. Baskes, S. G. Srinivasan, T. R. Cundari, A. K. Wilson, Modified embedded atom method study of the mechanical properties of carbon nanotube reinforced nickel composites, Phys. Rev. B 81 (10) (2010) 104103. doi:10.1103/PhysRevB.81.104103.

[46] P. Vashishta, R. K. Kalia, A. Nakano, J. P. Rino, Interaction potential for silicon carbide: A molecular dynamics study of elastic constants and vibrational density of states for crystalline and amorphous silicon carbide, J. App. Phys. 101 (10) (2007) 103515. doi:10.1063/1.2724570

[47] Y. Shi, A mimetic porous carbon model by quench molecular dynamics simulation, J. Chem. Phys. 128 (23) (2008) 234707. doi:10.1063/1.2943645.

[48] T. Kumagai, S. Hara, J. Choi, S. Izumi, T. Kato, Development of empirical bond-order-type interatomic potential for amorphous carbon structures, J. App. Phys. 105 (6) (2009) 064310. doi:10.1063/1.3086631.

[49] T. Liang, T.-R. Shan, Y.-T. Cheng, B. D. Devine, M. Noordhoek, Y. Li, Z. Lu, S. R. Phillpot, S. B. Sinnott, Classical atomistic simulations of surfaces and heterogeneous interfaces with the charge-optimized many body (COMB) potentials, Mater. Sci. Eng. R-Rep. 74 (9) (2013) 255-279. doi:10.1016/j.mser.2013.07.001.

[50] R. E. Franklin, Crystallite growth in graphitizing and non-graphitizing carbons, Proc. Roy. Soc. A 209 (1097) (1951) 196-218. doi:10.1098/rspa.1951.0197.

[51] A. V. Akimov, O. V. Prezhdo, Large-scale computations in chemistry: A bird's eye view of a vibrant field, Chem. Rev. 115 (2015) 57975890. doi:10.1021/cr500524c.

[52] N. Marks, Modelling diamond-like carbon with the environment-dependent interaction potential, J. Phys. Condens. Matter 14 (11) (2002) 2901-2927. doi:10.1088/0953-8984/14/11/308.

[53] M. Z. Bazant, E. Kaxiras, J. F. Justo, Environment-dependent interatomic potential for bulk silicon, Phys. Rev. B 56 (14) (1997) 8542-8552. doi:10.1103/PhysRevB.56.8542.

[54] J. F. Justo, M. Z. Bazant, E. Kaxiras, V. V. Bulatov, S. Yip, Interatomic potential for silicon defects and disordered phases, Phys. Rev. B 58 (5) (1998) 2539-2550. doi:10.1103/PhysRevB.58.2539.

[55] D. W. M. Lau, D. G. McCulloch, N. A. Marks, N. R. Madsen, A. V. Rode, High-temperature formation of concentric fullerene-like structures within foam-like carbon: Experiment and molecular dynamics simulation, Phys. Rev. B 75 (23) (2007) 233408. doi:10.1103/PhysRevB.75.233408.

[56] I. Suarez-Martinez, P. J. Higginbottom, N. A. Marks, Molecular dynamics simulations of the transformation of carbon peapods into double-walled carbon nanotubes, Carbon 48 (12) (2010) 3592-3598. doi:10.1016/j.carbon.2010.06.004.

[57] R. C. Powles, N. A. Marks, D. W. M. Lau, Self-assembly of $s p^{2}$-bonded carbon nanostructures from amorphous precursors, Phys. Rev. B 79 (7) (2009) 075430. doi:10.1103/PhysRevB.79.075430.

[58] T. P. Senftle, S. Hong, M. M. Islam, S. B. Kylasa, Y. Zheng, Y. K. Shin, C. Junkermeier, R. Engel-Herbert, M. J. Janik, H. M. Aktulga, T. Verstraelen, A. Grama, A. C. T. van Duin, The ReaxFF reactive force-field: development, applications and future directions, Nat. Computational Mater. 2 (2016) 15011. doi:10.1038/npjcompumats.2015.11.

[59] D. W. Brenner, The art and science of an analytic potential, Phys. Status Solidi B 217 (1) (2000) 23-40. doi:10.1002/(SICI)1521-3951(200001)217:1¡23::AID-PSSB23¡3.0.CO;2-N.

[60] H. M. Aktulga, J. C. Fogarty, S. A. Pandit, A. Y. Grama, Parallel reactive molecular dynamics: Numerical methods and algorithmic techniques, Parallel Computing 38 (4-5) (2012) 245-259. doi:10.1016/j.parco.2011.08.005.

[61] L. M. Ghiringhelli, J. H. Los, E. J. Meijer, A. Fasolino, D. Frenkel, Liquid carbon: structure near the freezing line, J. Phys.: Condens. Matter 17 (45) (2005) S3619-S3624. doi:10.1088/0953-8984/17/45/056.

[62] M. I. Katsnelson, A. Fasolino, Graphene as a prototype crystalline membrane, Acc. Chem. Res. 46 (1) (2013) 97-105. doi:10.1021/ar300117m.

[63] T. Liang, B. Devine, S. R. Phillpot, S. B. Sinnott, Variable charge reactive potential for hydrocarbons to simulate organiccopper interactions, J. Phys. Chem. A 116 (30) (2012) 7976-7991. doi:10.1021/jp212083t.

[64] N. A. Marks, Evidence for subpicosecond thermal spikes in the formation of tetrahedral amorphous carbon, Phys. Rev. B 56 (5) (1997) 2441-2446. doi:10.1103/PhysRevB.56.2441.

[65] G. Bussi, D. Donadio, M. Parrinello, Canonical sampling through velocity rescaling, J. Chem. Phys. 126 (1) (2007) 014101. doi: $10.1063 / 1.2408420$.

[66] D. S. Franzblau, Computation of ring statistics for network models of solids, Phys. Rev. B 44 (10) (1991) $4925-4930$. doi:10.1103/PhysRevB.44.4925.

[67] N. A. Marks, N. C. Cooper, D. R. McKenzie, D. G. McCulloch, P. Bath, S. P. Russo, Comparison of density-functional, tight-binding, and empirical methods for the simulation of amorphous carbon, Phys. Rev. B 65 (7) (2002) 075411. doi:10.1103/PhysRevB.65.075411.

[68] B. E. Warren, X-Ray diffraction, Addison-Wesley Publishing Co., Reading, Massachusetts, 1969.

[69] J. Schwan, S. Ulrich, H. Roth, H. Ehrhardt, S. R. P. Silva, R. J., et al., Tetrahedral amorphous carbon films prepared by magnetron sputtering and dc ion plating, J. App. Phys. 79 (3) (1996) 1416-1422. doi:10.1063/1.360979.

[70] D. G. McCulloch, D. R. McKenzie, C. M. Goringe, Ab initio simulations of the structure of amorphous carbon, Phys. Rev. B 61 (2000) 2349-2355. doi:10.1103/PhysRevB.61.2349.

[71] N. A. Marks, D. R. McKenzie, B. A. Pailthorpe, M. Bernasconi, M. Parrinello, Microscopic structure of tetrahedral amorphous carbon, Phys. Rev. Lett. 76 (5) (1996) 768-771. doi:10.1103/PhysRevLett.76.768.

[72] L. Li, M. Xu, W. Song, A. Ovcharenko, G. Zhang, D. Jia, The effect of empirical potential functions on modeling of amorphous carbon using molecular dynamics method, App. Surf. Sci. 286 (2013) 287-297. doi:10.1016/j.apsusc.2013.09.073.

[73] A. C. Ferrari, B. Kleinsorge, N. A. Morrison, A. Hart, V. Stolojan, J. Robertson, Stress reduction and bond stability during thermal annealing of tetrahedral amorphous carbon, J. Appl. Phys. 85 (1999) 7191. doi:10.1063/1.370531.

[74] F. P. Bundy, W. A. Bassett, M. S. Weathers, R. J. Hemley, H. U. Mao, A. F. Goncharov, The pressure-temperature phase and transformation diagram for carbon; updated through 1994, Carbon 34 (2) (1996) 141-153. doi:10.1016/0008- 
6223(96)00170-4.

[75] J. V. Badding, A. D. Lueking, Reversible high pressure $s p^{2}-s p^{3}$ transformations in carbon, Phase Transit. 80 (10-12) (2007) 1033-1038. doi:10.1080/01411590701473044.

76] Y. Lin, L. Zhang, H.-k. Mao, P. Chow, Y. Xiao, M. Baldini, J. Shu, W. L. Mao, Amorphous diamond: A high-pressure superhard carbon allotrope, Phys. Rev. Lett. 107 (17) (2011) 175504. doi:10.1103/PhysRevLett.107.175504.

[77] M. T. Yin, M. L. Cohen, Structural theory of graphite and graphitic silicon, Phys. Rev. B 29 (1984) 6996. doi:10.1103/PhysRevB.29.6996. 